\title{
Nanoparticles for light management in ultrathin chalcopyrite solar cells
}

\author{
Martina Schmid ${ }^{\text {a) }}$ \\ Nanooptische Konzepte für die PV, Helmholtz-Zentrum Berlin, 14109 Berlin, Germany; and Department of \\ Physics, Freie Universität Berlin, 14195 Berlin, Germany \\ Phillip Manley \\ Nanooptische Konzepte für die PV, Helmholtz-Zentrum Berlin, 14109 Berlin, Germany \\ Andreas Ott \\ Institut Weiche Materie und Funktionale Materialien, Helmholtz-Zentrum Berlin, 14109 Berlin, Germany \\ Min Song \\ Nanooptische Konzepte für die PV, Helmholtz-Zentrum Berlin, 14109 Berlin, Germany; and Department of \\ Physics, Freie Universität Berlin, 14195 Berlin, Germany \\ Guanchao Yin \\ Nanooptische Konzepte für die PV, Helmholtz-Zentrum Berlin, 14109 Berlin, Germany
}

(Received 25 May 2016; accepted 23 September 2016)

\begin{abstract}
We evaluate the potential of inserting metallic, metal-dielectric core-shell, and fully dielectric nanoparticles in ultrathin chalcopyrite solar cells to enhance absorption which experiences a significant drop for absorber thicknesses below $500 \mathrm{~nm}$. For different integration positions at the front or at the rear of the solar cell structure theoretical expectations and potential benefits originating from light scattering, near-field enhancement and coupling into waveguide modes by the nanoparticles are presented. These benefits are always balanced against experimental challenges arising for particular geometries due to the very specific fabrication processes of chalcopyrite solar cells. In particular high absorber deposition temperatures as well as contact layers that are relatively thick compared to other devices need to be considered. Based on this, we will need to go beyond some geometries that have proven beneficial for other types of solar cells and identify the most promising configurations for chalcopyrite-based devices.
\end{abstract}

\section{INTRODUCTION}

Chalcopyrite solar cells have recently reached a new record efficiency of $22.6 \%,{ }^{1}$ thus demonstrating the continuous improvement of this technology. Furthermore, $\mathrm{Cu}(\mathrm{In}, \mathrm{Ga}) \mathrm{Se}_{2}$ (CIGSe) polycrystalline thin-film solar cells stand out due to their high stability and tolerance against environmental influences e.g., shading or cosmic radiation. ${ }^{2}$ With an additional short energy payback time they constitute a serious thin-film competitor for wafer-based silicon solar cells. When envisaging a high production volume, however, the scarcity of the contained element indium can become a limiting factor. This consideration and the high supply risk of In [8.1/10 in 2015 (Ref. 3)] need to be addressed by a reduction of absorber material usage. Reduced material consumption will also help to further reduce costs, since material consumption still contributes to more than $60 \%$ of

\footnotetext{
Contributing Editor: Winston V. Schoenfeld

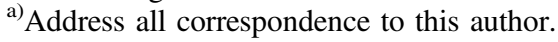

e-mail: martina.schmid@ @elmholtz-berlin.de

This paper has been selected as an Invited Feature Paper.

DOI: $10.1557 /$ jmr.2016.382
}

production costs. $^{4}$ When approaching the material reduction by reducing the absorber thickness from typical values of $2-3 \mu \mathrm{m}$ to below $500 \mathrm{~nm}$, a further benefit will lie in an increasing production throughput. Apart from these economic aspects, thin absorbers are beneficial for reduced recombination in the bulk. On the other hand, they bring the challenges of more serious interface recombination and in particular incomplete absorption. We will focus on the optical aspect and investigate how the absorption of ultrathin CIGSe solar cells can be enhanced by integrating nanoparticles.

Nanoparticles for absorption enhancement have been successfully applied in various types of solar cells and there are numerous examples for integration in amorphous silicon, crystalline silicon, or organic devices. ${ }^{5}$ Metal nanoparticles were initially in focus due to their plasmonic effects allowing for strong light interaction. ${ }^{6}$ Large-angle scattering, strong near-fields and coupling into waveguide modes are the prominent effects. ${ }^{7}$ Despite these advantages, the parasitic absorption of metal nanoparticles and their limited chemical stability are serious drawbacks which make dielectric particles favorable. ${ }^{8}$ These are characterized by the absence of parasitic absorption and 
high thermal stability (inorganic dielectrics). At the same time they also give rise to resonant modes like Mie resonances ${ }^{9}$ or whispering gallery modes. ${ }^{10}$ As a unison between metals and dielectrics, core-shell nanoparticles are an option to exploit beneficial effects from both materials. ${ }^{11}$

In this paper, we evaluate the potential of integrating metallic, metal-dielectric core-shell, and fully dielectric nanoparticles in ultrathin chalcopyrite solar cells for absorption enhancement. For different integration positions within the solar cell structure, theoretical expectations and potential benefits are presented. These are always balanced against experimental challenges arising for particular geometries due to the very specific fabrication processes of CIGSe solar cells. We will summarize the geometries which have proven beneficial for other types of solar cells and elaborate on why or why not they are equally applicable for chalcopyrite-based devices. In this way, we will identify the most promising configurations for ultrathin CIGSe solar cells.

\section{METHODS}

\section{A. Theoretical}

Numerical calculations were carried out using the finite-element method. This approach solves partial differential equations, here Maxwell's equations, using a variational approach and a set of basis functions which are locally defined on domain elements. The computational domain is divided into small, so called finite elements with sizes adjustable to precisely fit the shapes of the geometry. In our simulations, either isolated particles at interfaces or hexagonally arranged periodic particles in a chalcopyrite solar cell structure were investigated.

For the case of an isolated particle all outer boundaries were set to be perfectly matched layers (PML) and the scattered field was calculated. The absorption and scattering behavior is expressed in the parameters absorption crosssection $C_{\text {abs }}$, scattering cross-section in transmission $C_{\text {scaT }}$, and in reflection $C_{\mathrm{scaR}}$. These values have been normalized to the geometric cross-section of the particle given by $\pi r^{2}, r$ being the radius of the particle. For isolated nanoparticles additionally the angular distribution function (ADF) of the scattered light can be extracted from the far-field. To probe far-field properties, the absorption of the surrounding media was set to zero.

For the nanoparticles integrated into the solar cell stack, periodic boundary conditions were chosen on the sides of the hexagonal unit cell in which the nanoparticle was centered and PMLs were used in the incident and outgoing light directions. In this full field calculation transmission $T$, reflection $R$ and absorption $A_{\text {CIGSe }}$ were the parameters extracted. The pitch between the particles was varied by a multiple $m$ of the particle diameter with an additional $10 \mathrm{~nm}$ avoiding contact between particles. For the contour plots presenting $T, R$, or $A_{\text {CIGSe }}$ as a function of $m$ and $r$, the wavelength dependent curves were multiplied with the solar spectrum and the energy per photon and then integrated over the wavelength range from 300 to $1200 \mathrm{~nm}$ leading to values in units of short circuit current density $\left(\mathrm{mA} / \mathrm{cm}^{2}\right)$. The position of the nanoparticles was varied between different interfaces of the thin-film solar cell structure. In the case of integration at an interior interface, the thickness of the incorporating layer was adapted to compensate for the nanoparticle volume. The primary thin-film stack was air/ZnO:Al (AZO)/i-ZnO/ $\mathrm{CdS} / \mathrm{CIGSe} / \mathrm{Mo}$ from top to bottom with typical thicknesses of 240, 130, 50, and $400 \mathrm{~nm}$ for AZO, intrinsic $\mathrm{ZnO}(\mathrm{i}-\mathrm{ZnO}), \mathrm{CdS}$, and CIGSe for an ultrathin chalcopyrite solar cell. As an alternative to the opaque Mo back contact a transparent conductive oxide like $\operatorname{In}_{2} \mathrm{O}_{3}: \mathrm{Sn}$ (ITO) can be chosen. In this case the interface to the glass substrate needs to be considered as well.

Since nanoparticles prepared by chemical methods (see below) are most easily obtainable in a spherical shape, spheres were used in our basic theoretical investigations. For better comparability between the simulation results, this shape was maintained for the various integration positions despite the reality that deposition on a substrate will tend to distort the nanoparticle shape. We will briefly address the influence of contact angle; however, the focus is rather aimed at a fundamental theoretical understanding of the mechanisms, which are within a certain range largely independent for different contact angles.

Figures 1(a) and 1(b) show examples of the computational structure of an isolated nanoparticle at an interface and of a nanoparticle integrated into the solar cell structure, respectively. The software tools we used were JCMsuite $^{12}$ and Comsol Multiphysics. ${ }^{13}$ The refractive index data of the thin films were extracted from transmission and reflection measurements of individual layers using the software RefDex. ${ }^{14,15}$ For the metallic nanoparticles Ag as the most promising plasmonic material in the visible was chosen with refractive index data from Palik. ${ }^{16}$ For the dielectric nanoparticles a refractive index of 1.46 was set, which equally corresponds to polystyrene (PS) or $\mathrm{SiO}_{2}$. In the electric field maps shown throughout the paper, the plane of polarization is represented.

\section{B. Experimental}

The experimental structure of the chalcopyrite solar cell is $\mathrm{AZO} / \mathrm{i}-\mathrm{ZnO} / \mathrm{CdS} / \mathrm{CIGSe} / \mathrm{Mo} /$ glass. Also here ITO can be chosen as a transparent back contact replacing Mo. Mo or ITO was deposited by sputtering and had a thickness of $800 \mathrm{~nm}$ or $200 \mathrm{~nm}$, respectively. The ultrathin CIGSe absorber of less than $500 \mathrm{~nm}$ thickness was grown in a three-stage co-evaporation process at maximum temperatures between 440 and $600{ }^{\circ} \mathrm{C} .{ }^{17} \mathrm{CdS}$ 


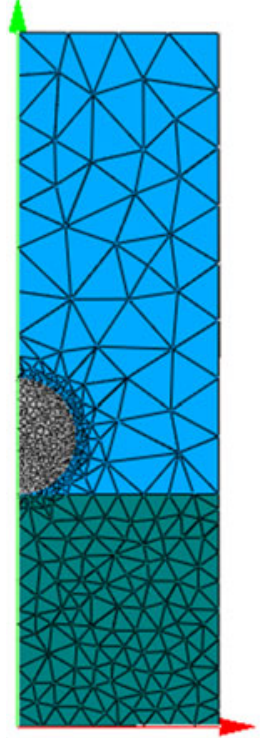

(a)

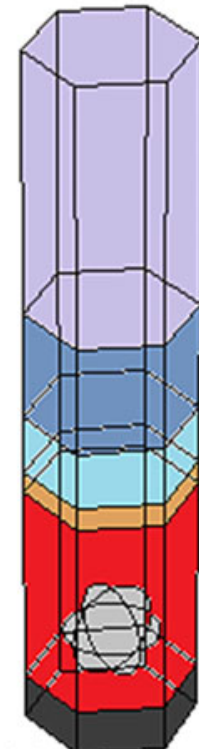

(b) air

AZO $\mathrm{i}-\mathrm{ZnO}$ $\mathrm{CdS}$ CIGSe NP Mo

FIG. 1. Simulation structure. (a) Nanoparticle at interface (exemplarily $\mathrm{Ag}$ nanoparticle in air to AZO) for calculation of absorption and scattering cross-sections-PML surrounding not shown (JCMsuite), (b) nanoparticle integrated into the CIGSe solar cell structure [exemplarily $\mathrm{SiO}_{2}$ nanoparticle (NP) in CIGSe to Mo] for extraction of absorption and reflection-PML top and bottom layer not shown (Comsol).

with a thickness of $50 \mathrm{~nm}$ was coated by chemical bath deposition, whereas $\mathrm{i}-\mathrm{ZnO}$ and $\mathrm{AZO}$ with thicknesses of 130 and $240 \mathrm{~nm}$, respectively, were again sputtered. Finally, Ni/Al grids were evaporated for front contacting and the solar cells were scribed to areas of $0.5 \mathrm{~cm}^{2}$.

For the fabrication of the nanoparticles, chemical methods were used as a basis. PS or $\mathrm{SiO}_{2}$ spheres were deposited by a self-assembly technique. ${ }^{18,19}$ For this approach, commercially available ${ }^{20}$ colloidal solutions of the spheres were suspended in butanol $\left(\mathrm{SiO}_{2}\right)$ or in ethanol mixed with 1 vol\% styrene (PS). The suspension was then brought onto a water surface under which the substrate was submerged. The spheres form a monolayer at the air-water interface, which can be transferred to the substrate by sucking out the water. A two-dimensional closely packed sphere array thus results.

The hexagonally arranged $\mathrm{PS}$ or $\mathrm{SiO}_{2}$ spheres can serve directly as a light managing structure. Alternatively, the PS sphere array can be utilized as a mask for material-in our case $\mathrm{Ag}$ - deposited on top and filling the gaps in between the spheres. After removing the spheres by toluol, Ag triangles remain which can further be transformed to spheres by annealing at $300{ }^{\circ} \mathrm{C}$ for $20 \mathrm{~min}$. The size and spacing of the Ag spheres can be tuned by the size of the template PS spheres and the thickness of the deposited Ag layer. Finally, the spacing between the PS spheres can also be altered by plasma etching in $\mathrm{O}_{2}$ atmosphere. The PS sphere templates can then be used to fabricate $\mathrm{SiO}_{2}$ networks. (Etching $\mathrm{SiO}_{2}$ spheres is in contrast not as easy to handle experimentally as etching PS spheres.)

$\mathrm{Au} @ \mathrm{SiO}_{2}$ core-shell nanoparticles were synthesized in a three-step process. Gold nanorods were synthesized via the seeded growth method. ${ }^{21}$ In short, small spherical seeds were formed and added into a growth solution containing the gold precursor $\left(\mathrm{HAuCl}_{4}\right)$, a mild reducing agent (L-ascorbic acid), and a stabilizing and structure directing surfactant, cetyltrimethylammonium bromide (CTAB). Excess $\mathrm{CTAB}$ was removed from the nanorod dispersion via centrifugation. A mesoporous silica shell was then formed via hydrolyzation and condensation reaction of tetraethoxysilane directly on the CTAB-stabilized gold nanorods at $\mathrm{pH} 10.8 .^{22}$ After the reaction, the core-shell nanoparticles were washed and redispersed in ethanol via centrifugation. A second dense silica shell was grown onto the mesoporous core-shell nanoparticles to fill the pores and achieve higher thermal stability of the nanorods via the Stöber method in an ethanol/water mixture. ${ }^{23}$ The core-shell nanoparticles were redispersed in ethanol and deposited onto the substrates using spin-coating.

The morphology of the thin-film solar cells and the nanoparticles was investigated by scanning-electron microscopy (SEM; Zeiss Leo Gemini 1530, Carl Zeiss AG, Oberkochen, Germany). X-ray photoelectronspectroscopy (XPS) was applied to probe diffusion of material. Optical measurements of transmission and reflection were carried out using a UV-Vis photospectrometer with an integrating sphere (PerkinElmer Lambda 950, PerkinElmer, Rodgau, Germany). Electrical characterization of the solar cells focused on the measurement of external quantum efficiencies (EQE). The EQE was taken in a two-source illumination system with a xenon and a halogen lamp; calibrated $\mathrm{Si}$ and Ge diodes were used as references.

\section{RESULTS AND DISCUSSION}

\section{A. Metallic nanoparticles}

\section{Integration at front}

\section{a. Theoretical}

$\mathrm{Ag}$ can be seen as the most promising plasmonic material for application in the visible wavelength spectrum. In contrast to materials such as $\mathrm{Au}$, it shows no interband transitions in that wavelength region and compared to non-noble metals like $\mathrm{Cu}$, it gives rise to higher scattering. ${ }^{24,25}$ Additionally, Ag nanoparticles are easy to prepare compared to other materials like $\mathrm{Al}$. Therefore, we perform our investigations of metallic nanoparticles on Ag-based ones.

The absorption and scattering cross-section of a nanoparticle are dependent on its radius, which can already be seen in the dipole approximation where $C_{\mathrm{abs}} \propto r^{3}$ and $C_{\text {sca }} \propto r^{6} .{ }^{26}$ These proportionalities imply that the scattering cross-section will increase more rapidly than 
the absorption cross-section for an increasing particle radius. Figure 2(a) visualizes the contribution of absorption and scattering to extinction cross-section $C_{\text {ext }}=C_{\text {sca }}+C_{\text {abs }}$ as a function of particle radius; in addition, scattering in the forwards $\left(C_{\text {scaT }}\right)$ and backwards $\left(C_{\text {scaR }}\right)$ direction are separated. A Ag nanoparticle surrounded by air and sitting on AZO was assumed, which corresponds to the front interface of the CIGSe solar cell. As can clearly be seen, the absorption dominates for small nanoparticles and becomes marginal starting from a radius of approximately $75 \mathrm{~nm}$. Absorption is assumed to be lost in the form of heat and therefore called parasitic absorption. A trend toward higher forward scattering can also be observed, yet the difference between $C_{\text {scaT }}$ and $C_{\text {scaR }}$ is not too distinct. Since for larger particles the center moves further from the interface, backwards scattering of the dipole mode can be enhanced. Note that the values presented were obtained by multiplying the cross-sections with the solar spectrum and integrating over the wavelengths from 300 to $1200 \mathrm{~nm}$.
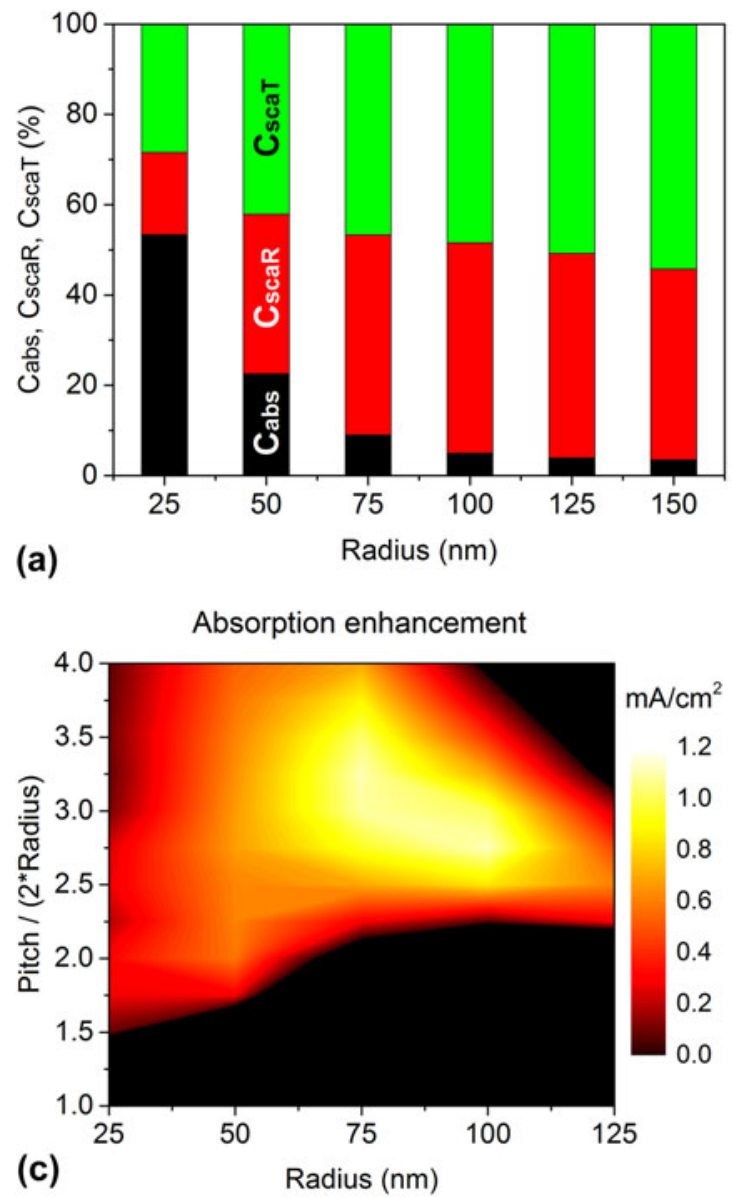

Examples of $C_{\text {scaT }}$ and $C_{\text {scaR }}$ as a function of wavelength are given in Fig. 2(b). Whereas for $25 \mathrm{~nm}$ nanoparticle radius the scattering cross-sections are almost negligible, they are significantly greater than unity for large radii. Scattering cross-sections larger than unity signify that the nanoparticle interacts with light from an incident area larger than its geometrical cross-section. This behavior allows for a reduced coverage whilst maintaining full light interaction. A low coverage is highly beneficial for metallic nanoparticles due to their parasitic absorption for small nanoparticles and increasing backwards scattering for larger ones. Looking for example at the $r=75 \mathrm{~nm}$ nanoparticle, $C_{\text {scaT }}=3$ is easily exceeded and we could imagine that a nanoparticle coverage of only one third of the surface area would be sufficient that all incident light will, on average, interact with a nanoparticle. In this case, however, $C_{\mathrm{scaR}}$ will not exceed 3 and therefore can be suppressed. When moving on to even larger particles, such as $r=125 \mathrm{~nm}$ as depicted further in Fig. 2(b), the peak broadens and the maximum scattering cross-section decreases, which

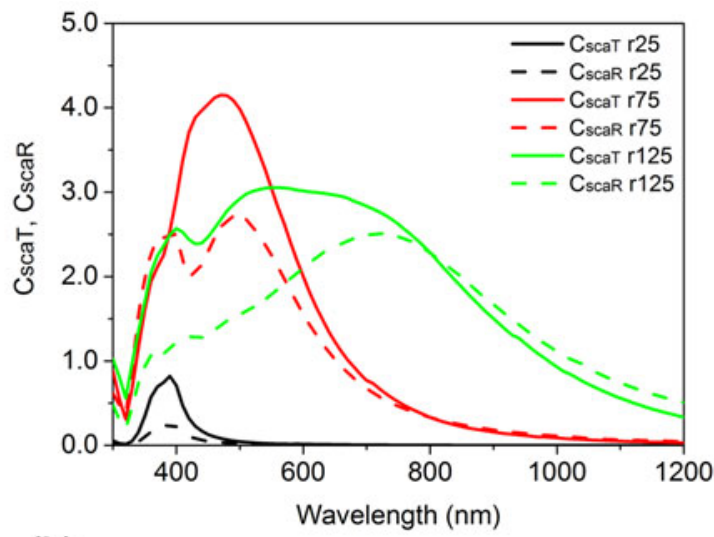

(b)

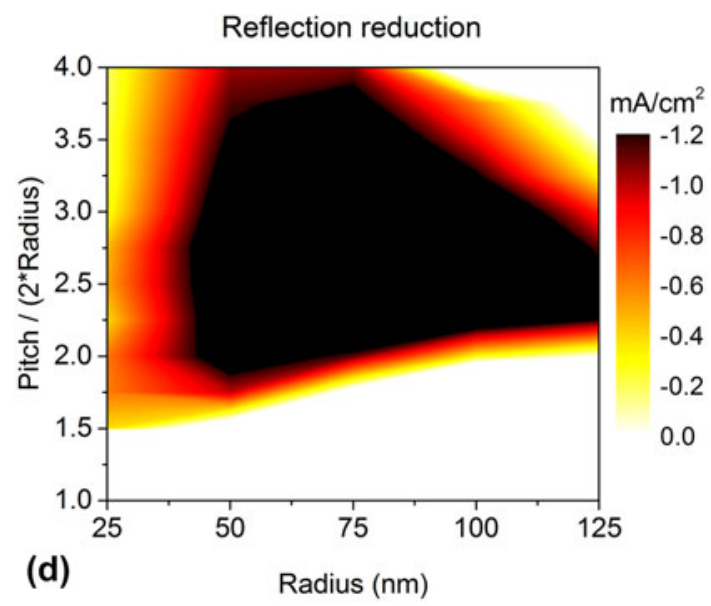

FIG. 2. Theoretical integration of metallic Ag nanoparticles at front (air/AZO) interface. (a) Absorption cross-section $C_{\text {abs }}$ and scattering crosssection in backward $\left(C_{\mathrm{scaR}}\right)$ and forward $\left(C_{\mathrm{scaT}}\right)$ direction for various radii, (b) $C_{\text {scaT }}$ (solid lines) and $C_{\text {scaR }}$ (dashed lines) as a function of wavelength for nanoparticle radii of 25,50 , and $75 \mathrm{~nm}$; (c) absorption enhancement in CIGSe as a function of radius and pitch as multiple of diameter, and (d) corresponding reduction in reflection. 
makes it more difficult to deduce a coverage for maximum interaction of $C_{\text {scaT }}$ but reduced contribution of $C_{\text {scaR }}$. These observations already point toward the fact that there will be a quite narrow optimum of particle size and coverage for which absorption enhancement can be obtained.

We now move on to consider the integration of the $\mathrm{Ag}$ nanoparticles at the front interface of our ultrathin CIGSe solar cell. For the sake of comparability, we continue to assume spherical particles on top of the front AZO layer, although in reality a certain deviation from the spherical shape and a larger contact angle are likely to exist. It should be pointed out that these deviations will influence the scattering curves and results differing to those presented here could easily be obtained for certain configurations. A match with experimental results and an absolute prediction of optimum parameters therefore will require detailed information about the realistic system. Here, we however seek to explain the basic effects that need to be taken into account and evaluate their impact, which is largely independent of contact angle. As we have seen from Fig. 2(a), on the one hand a minimum size of $\mathrm{Ag}$ nanoparticles is required for scattering to surpass absorption. On the other hand, drawbacks of larger $\mathrm{Ag}$ particles are more pronounced reflection losses which cannot be separated well from transmission by choice of coverage [Fig. 2(b)] and shifting of resonances out of the visible. Therefore, it is not surprising to find a maximum absorption enhancement in a relatively narrow window around the optimum radius and coverage. Figure 2(c) shows a contour image of absorption enhancement in the CIGSe layer as a function of nanoparticle radius and pitch as a multiple of the particle diameter. Pitch instead of coverage was chosen for sake of clarity; due to the dependence on particle radius the two parameters are not simply linearly related. The maximum enhancement of $1.2 \mathrm{~mA} / \mathrm{cm}^{2}$ is found for radii around $85 \mathrm{~nm}$ and a pitch to diameter ratio of 3 . These values match very well with the estimations we made based on calculations of single particles at the air-AZO interface. The low coverage enables the isolated treatment of the nanoparticles for good predictions.

In Fig. 2(d) the reduction in reflection corresponding to Fig. 2(c) is shown, also expressed in terms of short circuit current densities. The color scales are chosen to cover the same absolute range so that reduced reflection and increased absorption can be directly correlated. Notably, a reflection reduction of $1.2 \mathrm{~mA} / \mathrm{cm}^{2}$ can be obtained over a much wider parameter space than the absorption enhancement for the same value. This implies the existence of significant losses such that not all of the increase in light incoupling is translated into enhanced absorption in CIGSe. The main source of losses constitute the $\mathrm{AZO}, \mathrm{i}-\mathrm{ZnO}$, and $\mathrm{CdS}$ front contact layers. Due to their parasitic absorption, they will diminish the amount of light reaching the CIGSe absorber layer. Preferential large-angle scattering, which actually is seen as one of the major benefits of plasmonic nanoparticles, cannot be positively exploited: the enhanced path length in the front contact layers will rather increase their parasitic absorption, and as a result of Snell's law, the large scattering angles will have significantly lowered after the light has traversed many successive interfaces on the way to the highest index material CIGSe (for details see also Ref. 27). Based on these considerations, obtaining absorption enhancement by integrating metallic nanoparticles at the front interface of ultrathin CIGSe solar cells is highly challenging.

\section{b. Experimental}

Figure 3(a) shows in SEM pictures the experimental realization of $\mathrm{Ag}$ nanoparticles fabricated on top of ultrathin CIGSe solar cells. On the left, Ag triangles are visible which were obtained by depositing $250 \mathrm{~nm} \mathrm{Ag}$ on top of a $607 \mathrm{~nm}$ diameter PS sphere array before releasing the latter one. As can be seen, apart from triangles also some bowties remain. The right part of Fig. 3(a) gives the resulting nanoparticles after subjecting the triangles to a heat treatment at $200{ }^{\circ} \mathrm{C}$ for $1 \mathrm{~h}$. Spheres are formed with a radius of approx. $75 \mathrm{~nm}$ and a nearest-neighbor distance of $350 \mathrm{~nm}$ as resulting from the closely packed PS sphere array. Originating from the bowties, some large particle agglomerates remain and also some small residues around the nanoparticles can be found.

Figure 3(b) reveals at the top the reflection of the $\mathrm{Ag}$ triangles and spheres deposited for reference on a glass substrate. The resonance is clearly visible with the peak for the Ag triangles being more than $300 \mathrm{~nm}$ red-shifted compared to the $\mathrm{Ag}$ spheres. Following our theoretical investigations, we would expect these nanostructures to enhance the absorption in an underlying ultrathin CIGSe solar cell. However, the experimental result is not in agreement with these expectations: CIGSe solar cells with $\mathrm{Ag}$ nanostructures on top always suffer from deteriorated electrical properties. A detrimental influence of chemical and temperature treatment of the samples as a result of nanoparticle deposition could be excluded by reference experiments. The central drawback of the nanoparticle integration is revealed in the bottom graph of Fig. 3(b): with integrated $\mathrm{Ag}$ nanostructures on top, the overall reflection of the ultrathin CIGSe device increases following the resonant behavior of the nanostructures. Thus, the plasmonic resonance cannot be efficiently exploited for coupling light into the CIGSe solar cell structure and the small but theoretically present absorption enhancement cannot be observed.

Metallic nanoparticles added to the front of $\mathrm{GaAs}$ or $\mathrm{Si}$ solar cells had given the first proof of concept for absorption enhancement from plasmonic nanostructures. ${ }^{6,28,29}$ These 


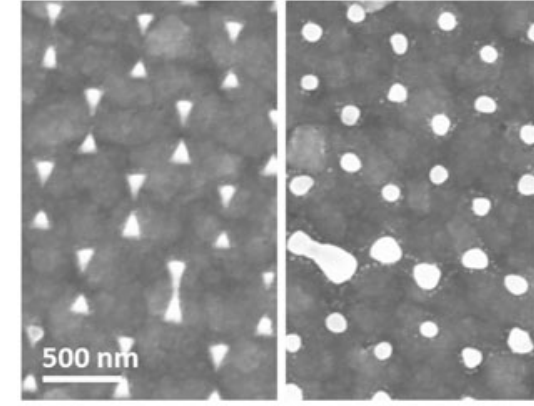

(a)

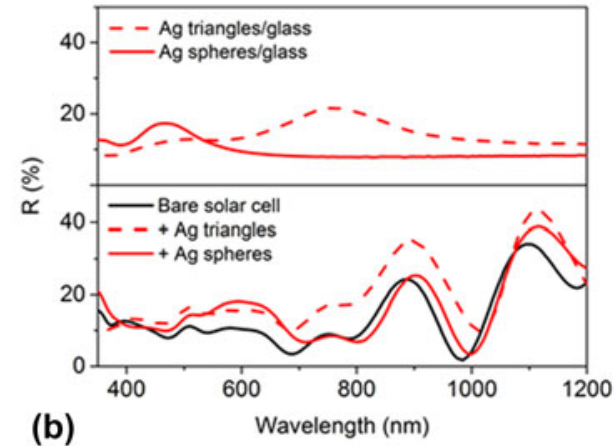

(b)

FIG. 3. Experimental integration of metallic Ag nanoparticles at front (air/AZO) interface. (a) SEM top view of Ag triangles (left), transformed to $\mathrm{Ag}$ nanoparticles (right) on top of a completed ultra-thin CIGSe solar cell; (b) reflection of (top) Ag triangles and spheres on a glass substrate and (bottom) of the same Ag nanostructures on top of the completed solar cell.

structures benefited from the proximity of the nanoparticles to the absorber layer with at maximum a spacer layer of a few tens of nanometers. Scattering and also near-field coupling could be directly exploited and losses minimized. In CIGSe solar cells, however, the thick front contact layers, totaling several hundred nanometers, separate the nanoparticles from the absorber. Losses are considerable and the positive impact of nanostructures on top of the completed device is significantly reduced.

\section{Integration at back}

\section{a. Theoretical}

The parasitic absorption and high reflectivity of metal nanoparticles suggests that integration at a rear interface of the solar cell structure may be more favorable. In this way, light will have once passed the absorber which will naturally filter out the short wavelengths from interacting with the nanoparticles. Thus, the losses in absorption by the nanoparticles can be suppressed. The reflectivity can be fully exploited since plasmonic particles are dominated by the electric dipole mode which scatters more strongly toward the material of higher refractive index. This can be understood via total internal reflection of scattered beams at the interface causing light to be trapped inside the higher refractive index material, which is formally covered by exploitation of the reciprocity theorem in Ref. 30.

Before looking into the scattering behavior of the $\mathrm{Ag}$ nanoparticles at the back, we need to decide which interface to choose. For full exploitation of scattering and a large angular distribution, a close contact to the absorber layer would be desirable. However, Ag can be built into the chalcopyrite structure forming (Ag, Cu) (In, Ga)Se 2 (Ref. 31) and therefore the nanoparticles are not considered to be stable in direct contact with the absorber. Thus, at least one intermediate layer should be present, which at the same time provides electrical passivation to avoid recombination at the metal surfaces. Mo is an opaque back contact- $\mathrm{Ag}$ nanoparticles underneath would not be of any use-but ITO as an alternative back contact can be chosen and the nanoparticles integrated thereunder.

Figure 4(a) shows $C_{\text {scaT }}$ and $C_{\text {scaR }}$ for an $r=75 \mathrm{~nm} \mathrm{Ag}$ nanoparticle in ITO sitting on the interface to glass (refractive index 1.5). Backwards scattering is strongly pronounced above a wavelength of $600 \mathrm{~nm}$. The insets of the ADF of the scattered light also indicate the high backwards fraction and scattering into high angle modes. For the nanoparticle at the front interface of air to AZO, the forward scattering was prominent. Now at the rear interface with inverted refractive index conditions, despite illumination from the same direction, backwards scattering is significant. As dipole scattering happens preferentially to the layer with the highest refractive index, which in our solar cell structure is the absorber, light scattering is always beneficially oriented. This scattering directivity toward higher index media is the most beneficial effect of metallic nanoparticles for our application.

Figure 4(b) plots the absorption enhancement of the CIGSe solar cell as a function of radius and pitch (multiple $m$ of diameter) of the Ag nanoparticles inserted underneath ITO. Compared to the reference solar cell on a transparent ITO back contact but without nanoparticles, absorption is increased up to $2.2 \mathrm{~mA} / \mathrm{cm}^{2}$ in the parameter range investigated and a maximum absolute value of $26.5 \mathrm{~mA} / \mathrm{cm}^{2}$ reached ( $r=50 \mathrm{~nm}, m=1)$; note that radii above $150 \mathrm{~nm}$ would not be feasible underneath an ITO layer of $250 \mathrm{~nm}$ thickness considering volume compensation but no conformal overgrowth. (250 nm ITO were chosen since we will see later that a $50 \mathrm{~nm}$ passivating layer with similar refractive index will need to be added to the $200 \mathrm{~nm}$ ITO.) The increase in absorption can be correlated to an increase in reflection [Fig. 4(c)] and a reduction in transmission [Fig. 4(d)]. Maximum reflection and absorption enhancement combined with highest transmission reduction are observed for the maximum coverage of closely packed nanoparticles. Compared to a reference with a planar Ag back reflector underneath ITO, which can reach a maximum of $27.1 \mathrm{~mA} / \mathrm{cm}^{2}$, these nanoparticle 

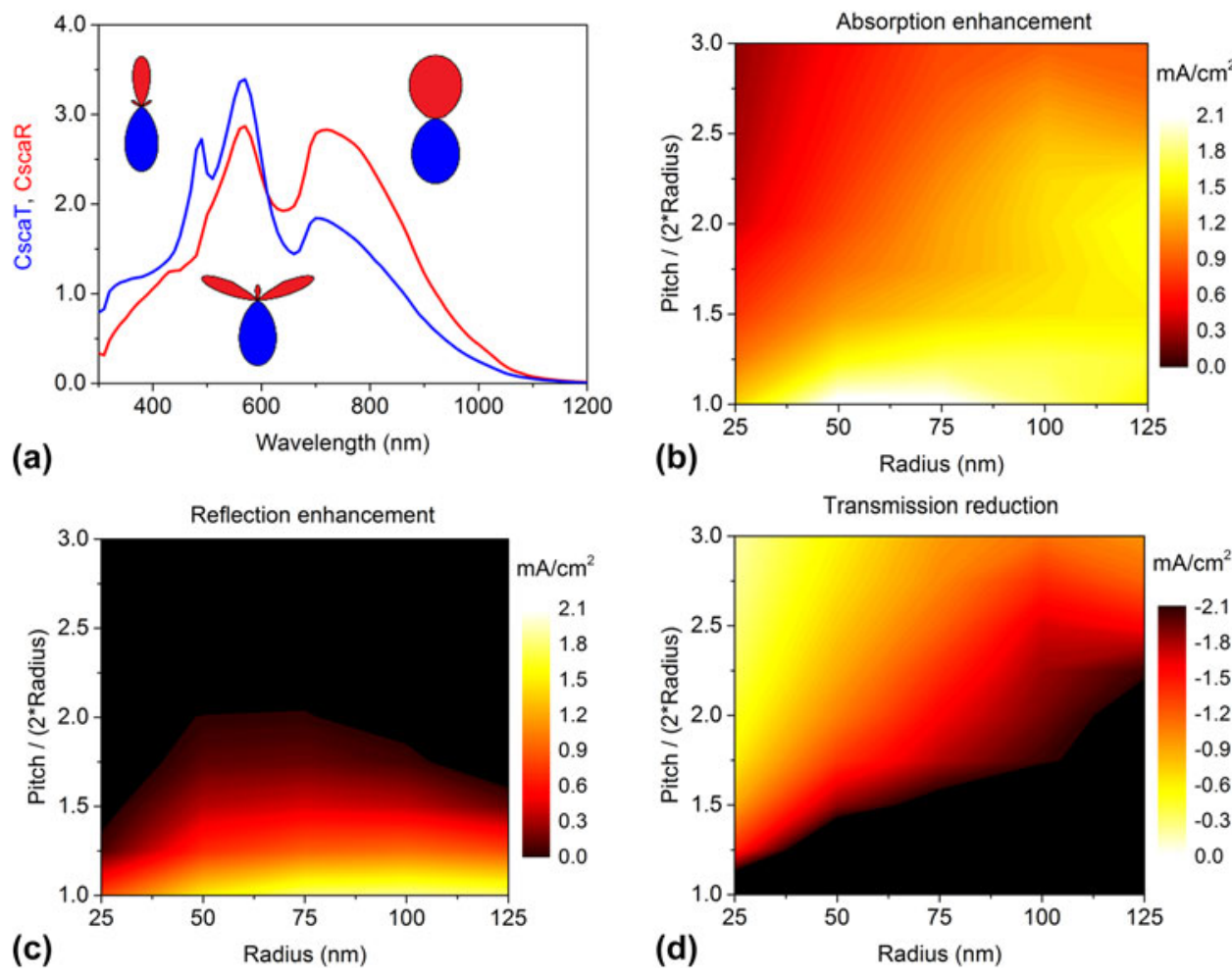

FIG. 4. Theoretical integration of metallic Ag nanoparticles at back (ITO/glass) interface. (a) Scattering cross-section in forwards $\left(C_{\mathrm{scaT}}\right.$, blue) and backwards $\left(C_{\mathrm{scaR}}\right.$, red) direction for an $r=75 \mathrm{~nm}$ nanoparticle, insets show angular scattering distribution for wavelengths of 490,570 and $710 \mathrm{~nm}$; (b) absorption enhancement in CIGSe as function of radius and multiple, (c) corresponding reflection enhancement, and (d) transmission reduction of the solar cell.

structures always provide less enhancement than the planar back reflector. On the other hand, they can provide transmission values coming close to the pure transparent back contact and which corresponds to $2.0 \mathrm{~mA} / \mathrm{cm}^{2}$ absolute in the case of $r=50 \mathrm{~nm}, m=1$. The sum of absorption and transmission of the solar cell structures with nanoparticles can significantly surpass the bare absorption with a back reflector underneath ITO since reflection is reduced. The large angular distribution of the light scattered back from the nanoparticles is beneficial to a certain extent, although diminished again due to the interlayer. By adjusting the coverage, the nanoparticles offer the opportunity to carefully tune absorption and transparency of the device, making it adaptable for configurations like tandem solar cells or semitransparent solar cells. In the case of a single junction device, transmitted light can also be exploited using an additional back reflector at the rear interface to scatter light back into the structure.

\section{b. Experimental}

Looking at the experimental realization of $\mathrm{Ag}$ nanoparticles integrated underneath the transparent ITO back contact, thermal stability is the key challenge. To test whether diffusion is blocked, Ag nanoparticles of approx.
$200 \mathrm{~nm}$ major diameter on a glass substrate (experimentally, the nanoparticles are more hemispherical and not symmetric) were overcoated with $200 \mathrm{~nm}$ ITO. The sample was then exposed to a CIGSe absorber growth process, thus experiencing the full high temperature run, but material deposition was avoided using a mask. The maximum process temperature was reduced from the standard 610 to $440{ }^{\circ} \mathrm{C}$ to minimize thermal impact. The process temperature cannot be reduced below $440{ }^{\circ} \mathrm{C}$ without compromising the device efficiency. ${ }^{17}$ Despite this temperature reduction, according to the XPS measurements presented in Fig. 5(a) (red curve), Ag is still found on the ITO surface after the process and diffusion could not be avoided.

Since a further reduction of process temperature is not an option, an additional diffusion blocking layer was inserted between the nanoparticles and the ITO. $\mathrm{Al}_{2} \mathrm{O}_{3}$ was chosen due to a similar refractive index as ITO, low parasitic absorption, and known use as passivation layer. In the first attempt, $150 \mathrm{~nm}$ sputtered $\mathrm{Al}_{2} \mathrm{O}_{3}$ was used, but no passivation was found. Since atomic layer deposition (ALD) is known to form highly compact layers, next $150 \mathrm{~nm}$ of $\mathrm{Al}_{2} \mathrm{O}_{3}$ was deposited via ALD. In this case, the Ag diffusion could be blocked and no signal was detected via XPS. Due to the high material quality, the thickness of ALD $\mathrm{Al}_{2} \mathrm{O}_{3}$ could even be reduced to $50 \mathrm{~nm}$ 

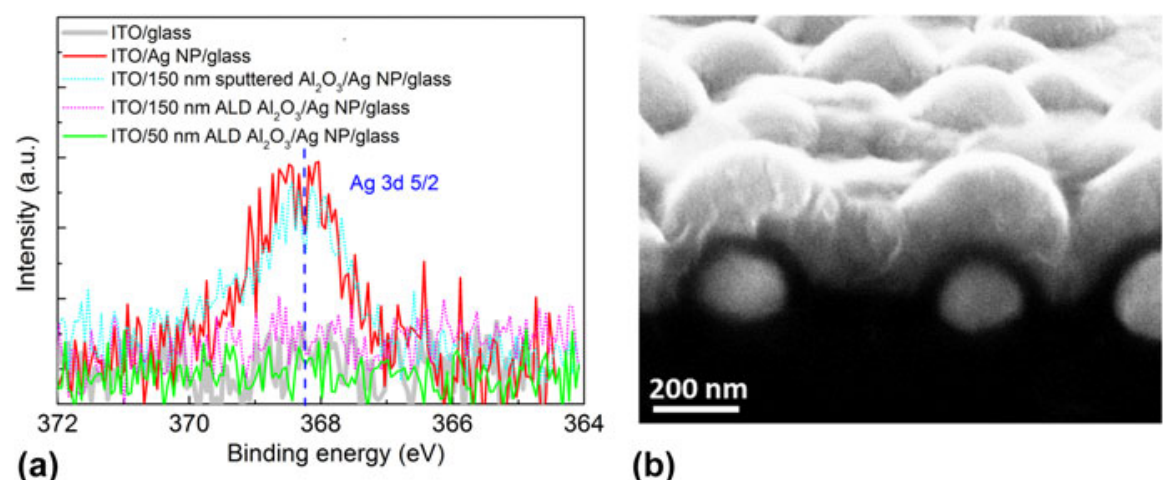

(b)

FIG. 5. Experimental integration of metallic Ag nanoparticles at back (ITO/glass) interface. (a) XPS measurements probing diffusion of Ag through ITO or ITO plus various passivating layers during a high temperature absorber deposition process; (b) SEM cross-section of thermally stabilized $\mathrm{Ag}$ nanoparticles underneath $50 \mathrm{~nm}$ ALD $\mathrm{Al}_{2} \mathrm{O}_{3}$ and $200 \mathrm{~nm}$ ITO. ${ }^{17}$

while maintaining the thermal passivation of the $\mathrm{Ag}$ nanoparticles [compare the green curve in Fig. 5(a)].

Figure 5(b) shows the cross-sectional SEM image of the successful structure ITO/50 nm ALD $\mathrm{Al}_{2} \mathrm{O}_{3} / \mathrm{Ag}$ nanoparticles/glass. A further experimental challenge arises from the fact that the required high coverage with Ag nanoparticles makes the back contact mechanically fragile. Scribing of the absorber for separating individual solar cells can easily delaminate the ITO back contact which due to the nanoparticles has a low adhesion to the substrate. An experimental proof of absorption enhancement using $\mathrm{Ag}$ nanoparticles underneath the transparent back contact of an ultrathin CIGSe solar cell is therefore challenging. Enhanced absorption could be found in optical measurements, the electrical proof is still lacking. More details can be found in Ref. 32.

In other technologies, in particular Si photovoltaics, the integration of $\mathrm{Ag}$ nanoparticles at the rear contact of the solar cell has been proven to be positive for absorption enhancement. The superstrate configuration in $\mathrm{c}-\mathrm{Si}^{33}$ or the lower process temperatures for a-Si deposition than for $\mathrm{CIGSe}^{34,35}$ facilitate a stable integration of the $\mathrm{Ag}$ nanoparticles. Only a thin electrically passivating layer is required to separate the nanoparticles from the absorber layer. Thus, scattering, near-field enhancement and in the case of regular particle arrangements also coupling to waveguide modes can be exploited effectively. For CIGSe solar cells, electrically and thermally stable integration of nanoparticles underneath the absorber resulted in a relatively thick interlayer system. Therefore, after successful stabilization, effective coupling of light back to the absorber remains the central challenge.

\section{B. Core-shell nanoparticles}

\section{Theoretical}

In the previous section we have looked at Ag nanoparticles integrated at the rear of a CIGSe solar cell. Due to the challenge of Ag diffusion into the absorber material at high process temperatures, the nanoparticles were inserted underneath the ITO transparent back contact and even an additional $\mathrm{Al}_{2} \mathrm{O}_{3}$ passivating layer was required to thermally stabilize them. In contrast, a close contact to the absorber layer would make it possible to fully benefit from the large-angle scattering and the enhanced near-field of the nanoparticles. Therefore, thermally but also electrically well-passivated metallic nanoparticles directly integrated into the absorber layer would be advantageous. This leads us to the consideration of metal-dielectric core-shell nanoparticles.

Figure 6(a) shows in black the scattering of an $r=75 \mathrm{~nm}$ nanoparticle in CIGSe and at the interface to Mo. The high-index surrounding has strongly red-shifted the resonances [compared to that of Fig. 4(a)], but the scattering cross-sections are equally high. Now we aim at maintaining the high scattering whilst electrically and thermally passivating the Ag nanoparticles in the absorber layer. Core-shell nanoparticles with a metal core and a dielectric shell are widely used for various applications. $\mathrm{SiO}_{2}$ is a very common shell material, thus we firstly assume a $\mathrm{Ag} @ \mathrm{SiO}_{2}$ nanoparticle with a shell thickness $t=10 \mathrm{~nm}$. The resulting scattering cross-section is plotted in red in Fig. 6(a): resonances are blue-shifted and the maximum value has dropped from approximately 4 to less than 2. Integration of these nanoparticles does not appear promising due to the poor optical properties. The reason for the scattering reduction of the $\mathrm{Ag} @ \mathrm{SiO}_{2}$ nanoparticles can be seen in the low refractive index of the shell material as compared to the surrounding CIGSe. As explained in Ref. 36, the low index shell traps the light in its inside and outwards scattering and fields are strongly suppressed. The solution is to choose a medium with a high refractive index as shell material. Since CIGSe has a refractive index of approximately 3 , we choose a constant refractive index of 4 for the shell material to exemplify this idea. The green curve in Fig. 6(a) shows the scattering of the Ag@n=4 (Ag@4) nanoparticle, with the shell thickness kept at $10 \mathrm{~nm}$. Resonances red-shift by approximately $100 \mathrm{~nm}$ compared 

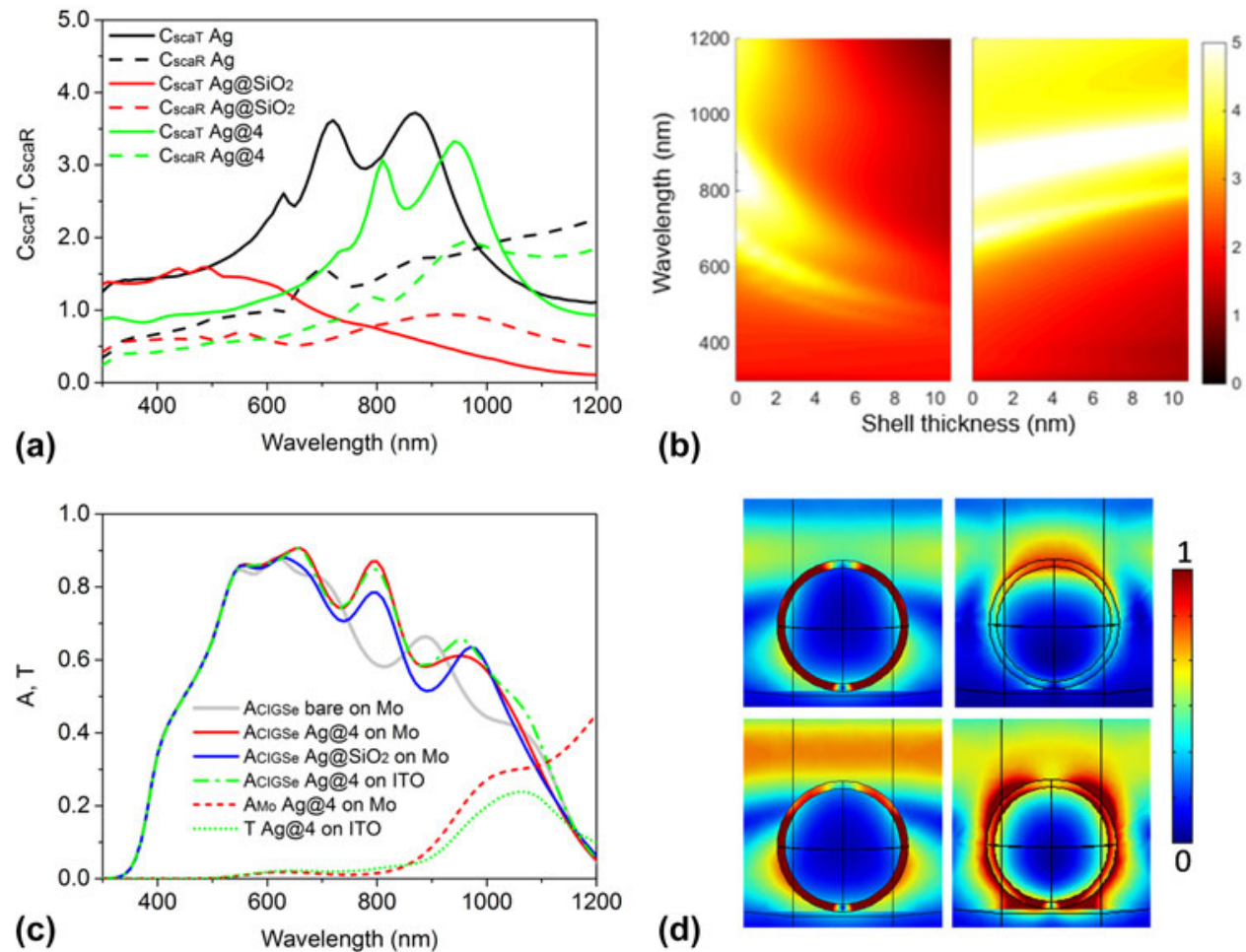

(d)

FIG. 6. Theoretical integration of core-shell nanoparticles at back (CIGSe/contact) interface. (a) Scattering cross-section in forward $\left(C_{\text {scaT }}\right)$ and backward $\left(C_{\mathrm{scaR}}\right)$ direction for an $r=75 \mathrm{~nm}$ bare $\mathrm{Ag}$ nanoparticle, a $\mathrm{Ag} @ \mathrm{SiO}_{2}$ and a $\mathrm{Ag} @ 4$ (10 nm shell thickness) core-shell nanoparticle in CIGSe to Mo; (b) $C_{\mathrm{sca}}$ as a function of shell thickness and wavelength for $\mathrm{SiO}_{2}$ (left) and $n=4$ shell (right) around the Ag nanoparticle in homogeneous CIGSe; (c) absorption in CIGSe for the Ag@ $\mathrm{SiO}_{2}$ and the Ag@ 4 core-shell nanoparticles integrated in CIGSe to Mo and the Ag@4 particles in CIGSe to ITO as compared to the bare solar cell, absorption in Mo $\left(A_{\mathrm{Mo}}\right)$, and transmission $T$ are given for the case of Ag@ 4 nanoparticles on Mo and ITO, respectively; (d) electric field distribution for $\mathrm{Ag} @ \mathrm{SiO}_{2}$ (left column) and $\mathrm{Ag}$ @ 4 (right column) core-shell nanoparticles in the solar cell structure for $\lambda=800 \mathrm{~nm}$ (top row) and $\lambda=950 \mathrm{~nm}$ (bottom row).

to the bare nanoparticle, and scattering efficiency is only slightly reduced. These nanoparticles appear highly promising for direct integration and absorption enhancement in CIGSe.

To investigate the influence of shell thickness, Fig. 6(b) plots the scattering cross-sections of $\mathrm{Ag} @ \mathrm{SiO}_{2}$ (left) and $\mathrm{Ag} @ 4$ (right) nanoparticles in a homogeneous CIGSe surrounding as a function of wavelength and shell thickness. For the $\mathrm{Ag} @ \mathrm{SiO}_{2}$ nanoparticles, scattering is essentially repressed when the shell thickness reaches $3 \mathrm{~nm}$, whereas for Ag@4 nanoparticles, scattering is maintained up to approx. $t=10 \mathrm{~nm}$. Since a $3 \mathrm{~nm}$ shell is not expected to provide any thermal passivation, this further supports the claim that a $\mathrm{SiO}_{2}$ shell will not be beneficial.

Next, we look at the results of integrating core-shell nanoparticles at the CIGSe/Mo interface of the complete solar cell structure. A minimum pitch was identified as ideal for the $r=75 \mathrm{~nm} \mathrm{Ag}$ nanoparticles in a $10 \mathrm{~nm}$ shell of refractive index 4. In the following, we focus on a multiple of 1.25 times the diameter. Figure 6(c) compares the absorption in CIGSe for that case (red) to the absorption without nanoparticles (gray). An increase of $1.6 \mathrm{~mA} / \mathrm{cm}^{2}$ short circuit current density is observed. For the same nanoparticles but with a $\mathrm{SiO}_{2}$ shell, the increase would only be $0.6 \mathrm{~mA} / \mathrm{cm}^{2}$, see the blue curve. The peak positions with integrated nanoparticles are at the same wavelengths, because they are only related to the CIGSe film thickness which is changed for volume compensation. Yet, the enhancement is lower for the lowindex shell in the entire wavelength range from 600 to $950 \mathrm{~nm}$. As we have seen for the Ag nanoparticles underneath ITO, a transparent back contact allows for a reduction in Mo parasitic absorption and an excellent balance between absorption enhancement and transparency. Therefore, in Fig. 6(c) we further show the case of Ag@4 nanoparticles on an ITO back contact and present absorption in CIGSe and transmission of the device (green). (The ITO thickness was set to $250 \mathrm{~nm}$ for better comparability to Ag nanoparticles underneath the back contact.) Absorption enhancement corresponding to a short circuit current density of $1.9 \mathrm{~mA} / \mathrm{cm}^{2}$ is even better than that for the situation on Mo back contact. In addition to that a transparency corresponding to $2.7 \mathrm{~mA} / \mathrm{cm}^{2}$ can be exploited. The absorption losses in Mo (red-dashed curve), equal to $3.8 \mathrm{~mA} / \mathrm{cm}^{2}$, mostly translated to transmission. This transmission can be exploited in a semitransparent device or be reflected back into the structure for further absorption enhancement. 
Finally, in Fig. 6(d) we present the electric field distributions around the core-shell nanoparticles for the $\mathrm{SiO}_{2}$ shell (left) and the refractive index 4 shell (right) for $\lambda=800 \mathrm{~nm}$ (top) and $\lambda=950 \mathrm{~nm}$ (bottom) in the solar cell structure. It is clearly revealed how the low-index shell traps the light, whereas the high-index one promotes the field propagation. ${ }^{36}$

\section{b. Experimental}

Similarly to the case of metallic nanoparticles integrated underneath an ITO back contact, also for the metal@dielectric core-shell particles thermal passivation has to be proven experimentally. Typically used core-shell nanoparticles are $\mathrm{Au} @ \mathrm{SiO}_{2}$, which are commonly accessible in the experiment. Therefore in contrast to the theoretical studies performed consistently for $\mathrm{Ag}$, we chose $\mathrm{Au}$ as core material and $\mathrm{SiO}_{2}$ for the shell in the basic experimental studies of diffusion. Only if diffusion through thin shell materials can be handled at all, will it be worth to seek out more optically suitable shell materials. Figure 7(a) shows the SEM cross-section of the $\mathrm{Au} @ \mathrm{SiO}_{2}$ nanoparticles underneath an ultrathin CIGSe absorber layer. In the inset, a TEM view of the nanoparticles is presented. It clearly reveals the core-shell structure of the ellipsoidal nanoparticles with a maximum diameter of approx. $50 \mathrm{~nm}$.

The visual presence of the nanoparticles under the absorber is already a good indication for their preservation. To investigate diffusion of the metal into and through the absorber layer, again XPS measurements were performed. Figure 7(b) summarizes the results: Whereas for the high process temperature of $610{ }^{\circ} \mathrm{C}$, $\mathrm{Au}$ is observed on top of the CIGSe layer; for lower temperatures of 440 and still $510{ }^{\circ} \mathrm{C}$, Au does not diffuse through the absorber. In these cases the particles can be assumed stable. For the experimental realization of the theoretically predicted promising structures, core-shell nanoparticles like Ag@AlSb would be desirable. These however pose the next challenge since the fabrication of a high refractive index dielectric shell is not a commonly established method. An alternative approach might consist in forming the shell from amorphous $\mathrm{Si}$ as it is a very commonly used semiconductor offering a high refractive index and a good transparency at least above its band gap of $1.7 \mathrm{eV}$. Judging from the highly promising properties of metal nanoparticles with a high refractive index shell and the potential of broader application, further research may be desirable.

Therefore, also for the optically effective integration of core-shell nanoparticles, CIGSe solar cells pose severe challenges. The beneficial integration of $\mathrm{Au} @ \mathrm{SiO}_{2}$ (Ref. 37) and Au@ $\mathrm{PVP}^{38}$ nanoparticles has been proven in low refractive index organic or dye sensitized solar cells, respectively. In the case of successful application of metal-insulator core-shell nanoparticles in the high refractive index perovskite absorber layer, effects beyond pure optical benefits are claimed to be the reason for enhancement. ${ }^{39}$ From the optical point of view we thus underline the need of high refractive index shell material to effectively exploit optical effects from core-shell nanoparticles in CIGSe solar cells.

\section{Dielectric nanoparticles}

\section{Integration at front}

\section{a. Theoretical}

Plasmonic metal nanoparticles revealed two fundamental drawbacks: parasitic absorption and low thermal stability. To avoid these negative influences, we turn instead to photonic nanoparticles made from dielectrics. They can provide scattering cross-sections equally high as metallic nanoparticles ${ }^{40,41}$ due to the presence of resonant modes. As the dielectric nanoparticles are free from parasitic absorption, we can easily place them on top of the finished solar cell to exploit their light coupling behavior. In Fig. 8(a) the absorption enhancement resulting from $\mathrm{SiO}_{2}$ sphere arrays is mapped for various

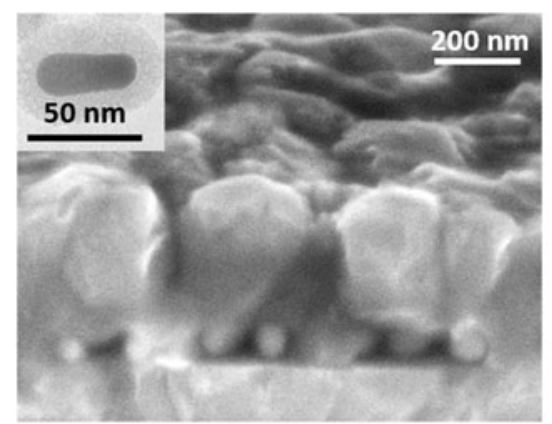

(a)

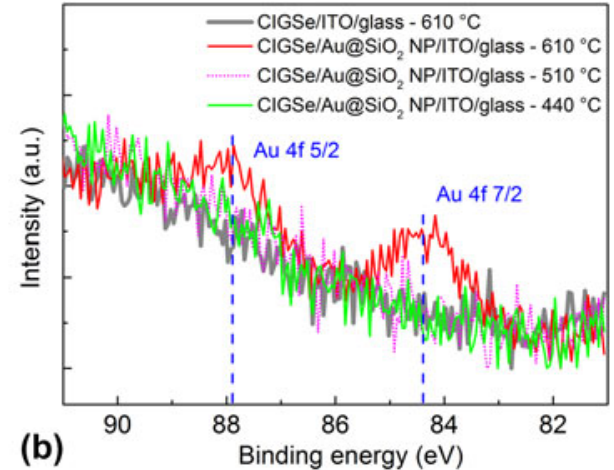

(b)

FIG. 7. Experimental integration of core-shell nanoparticles at back (CIGSe/contact) interface. (a) $\mathrm{Au} @ \mathrm{SiO}_{2}$ core-shell nanoparticles underneath a CIGSe absorber layer, inset shows TEM of bare core-shell nanoparticle; (b) XPS measurement probing diffusion of $\mathrm{Au} \mathrm{Xrough}_{\mathrm{SiO}}$ shell for various process temperatures of CIGSe deposition. 
nanoparticle radii and pitches. Closely packed arrays $(m=1)$ are the most beneficial with a trend toward slightly increased spacing $(m=1.25)$ for larger radii. Two distinct maxima can be observed: one for smaller radii of 60-75 nm, the other one for radii of $250 \mathrm{~nm}$ and above. These two cases will be discussed in the following.

For the smaller spheres, the peak in absorption enhancement corresponding to $1.6 \mathrm{~mA} / \mathrm{cm}^{2}$ comes with a clear reduction in reflection, compare Fig. 8(b). Actually, these particles are too small to show Mie resonances in the visible wavelength range which would lead to strong light interaction. For comparable resonance wavelengths, the dielectric nanoparticles generally need to be significantly larger than the metallic ones, see also Ref. 40. Therefore, the origin of the pronounced reduction in reflection cannot lie in resonant behavior. The closely packed nanoparticle array of $r=75 \mathrm{~nm} \mathrm{SiO}_{2}$ spheres with refractive index $n=1.46$ surrounded by air has an effective refractive index $n_{\text {eff }}=1.24$. The thickness of this effective index layer is $d_{\text {eff }}=150 \mathrm{~nm}$, which fulfills the $\lambda /(4 n)$ condition for $\lambda=744 \mathrm{~nm}$. $n_{\text {eff }}$ is close to the ideal value $n_{\text {ideal }}=\left(n_{1} \times n_{2}\right)^{1 / 2}=1.35$ for the refractive index of the intermediate antireflection layer between air $\left(n_{1}=1\right)$ and AZO $\left(n_{2}=1.82 @ 744 \mathrm{~nm}\right)$. The ideal thickness in this case would be $d_{\text {ideal }}=138 \mathrm{~nm}$, which is also very comparable to the effective thickness of nanospheres in air. Figure 8(c) compares the reflection of the ultrathin CIGSe solar cells with the $75 \mathrm{~nm}$ radius $\mathrm{SiO}_{2}$ sphere array on top (black line) to the one with the effective (green dashed) and the one with the ideal (purple dash-dotted) antireflection layer. They are very similar and all show a strong reduction in comparison to the cell without an antireflection coating (gray reference line). As the "ideal" antireflection layer was calculated for the interface air/AZO only, the nanosphere antireflection layer can reduce the reflection even more and another advantage lies in its increased acceptance angle. Whereas for planar layers, reflection can increase drastically with nonperpendicular incidence, the nanospheres take in light equally well from a wide angular range and for certain cases reveal even more pronounced field enhancements than for normal incidence. ${ }^{10}$ The reduction in reflection directly translates to an increase in absorption as the curves in Fig. 8(c) show.

Moving on to the larger spheres, the increase in absorption is not as pronounced, but becomes significant for spheres above $200 \mathrm{~nm}$ radius [Fig. 8(a)]. Here, Mie resonance can again be observed for visible wavelengths.
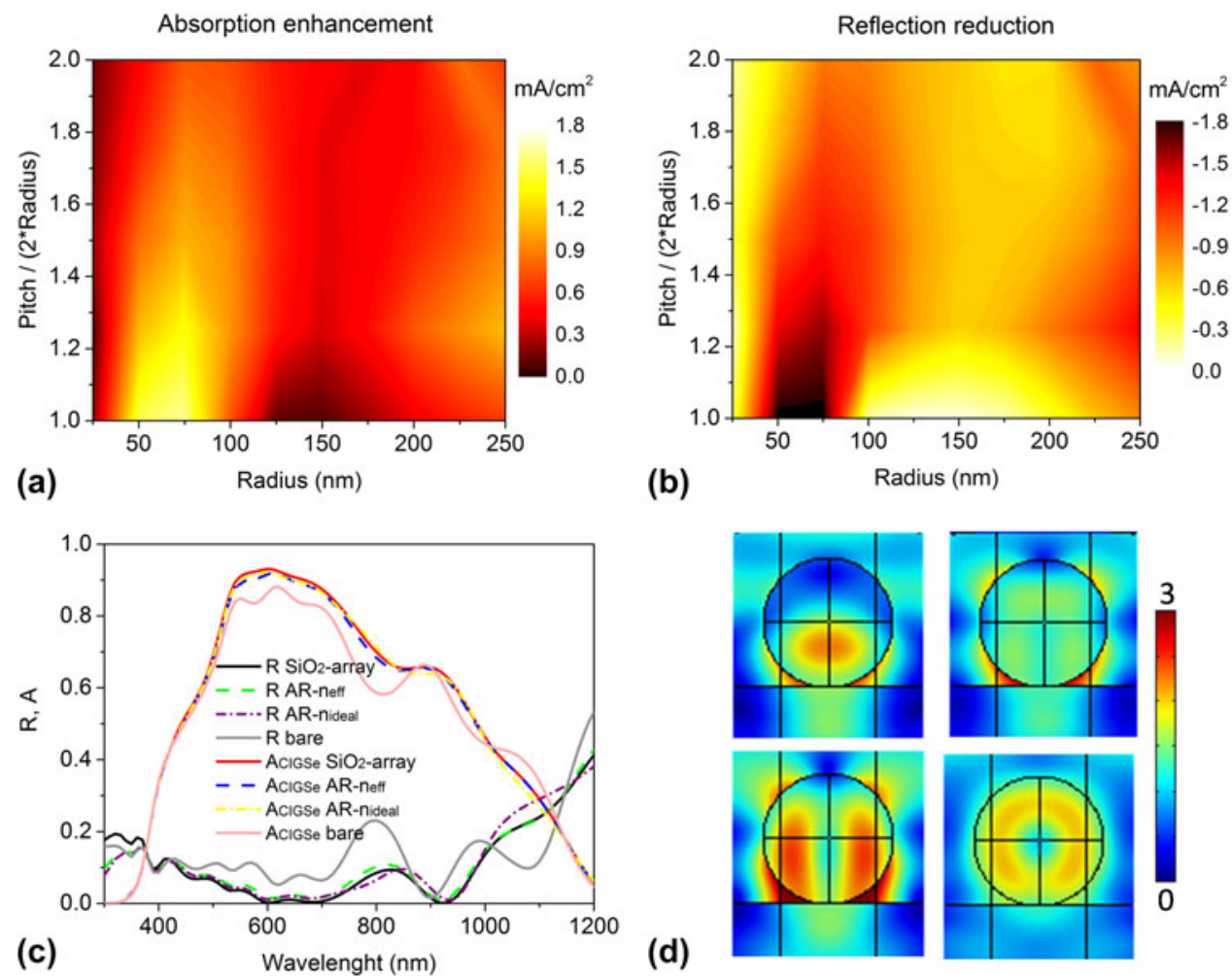

(d)
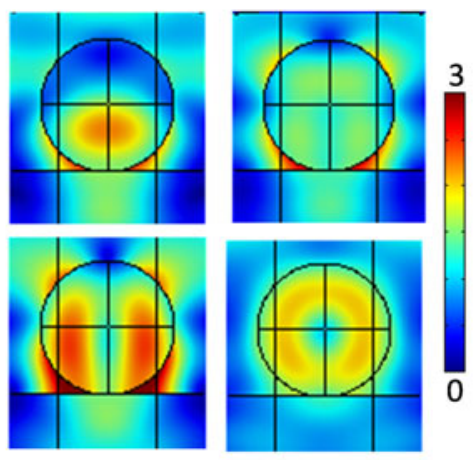

FIG. 8. Theoretical integration of dielectric $\mathrm{SiO}_{2}$ nanoparticles at front (air/AZO) interface. (a) Absorption enhancement in CIGSe as function of

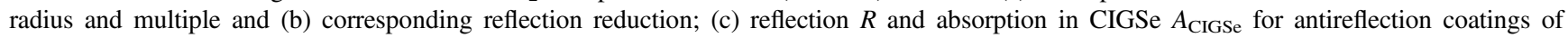
$r=75 \mathrm{~nm}$ closely packed $\mathrm{SiO}_{2}$ nanospheres $\left(\mathrm{SiO}_{2}\right.$-array), a $d=150 \mathrm{~nm}$ and $n_{\text {eff }}=1.24$ layer (AR- $\left.n_{\text {eff }}\right)$ and a $d=138 \mathrm{~nm}$ and $n_{\text {ideal }}=1.35$ layer (AR- $n_{\text {ideal }}$ ) as compared to the bare cell; (d) electric field distributions around $r=250 \mathrm{~nm}$ spheres for wavelengths $610 \mathrm{~nm}$ (top left), $640 \mathrm{~nm}$ (top right), $670 \mathrm{~nm}$ (bottom left) and $700 \mathrm{~nm}$ (bottom right). 
Due to the large particle size a multitude of modes occurs and since we are dealing with dielectrics, electric and magnetic modes are equally strongly pronounced. ${ }^{40,42}$ The Mie resonances of the dielectric nanoparticles always reveal a strong forward scattering or even focusing behavior. ${ }^{43}$ This effect can be seen in the electric field representation of the $r=250 \mathrm{~nm}$ nanospheres on top of the solar cell structure (excerpt of nanoparticles in air on AZO shown) for the wavelengths 610,640 , and $670 \mathrm{~nm}$ [Fig. 8(d) top left, top right and bottom left]. A strong transition between different modes even within this short wavelength interval is observed. At $\lambda=700 \mathrm{~nm}$, still another mode occurs which can be deduced to be a whispering gallery mode based on the field pattern [Fig. 8(d) bottom right]. ${ }^{44}$ High electric field intensities following the inner surface of the nanosphere are revealed. Thus, the large dielectric nanoparticles have a strong light interaction and the various modes can help to improve the performance of an underlying solar cell. Similar to the metallic nanoparticles, losses and incomplete light penetration down to the absorber layer need to be considered here as well. Therefore, as a conclusion for dielectric nanoparticles at the front interface, the antireflection effect of the small dielectric nanospheres can be seen as the most beneficial.

\section{b. Experimental}

Figure 9 presents the experimental results of depositing dielectric nanoparticles at the front interface of ultrathin CIGSe solar cells. Measurements of quantum efficiencies are given, while the insets show SEM images of the closely packed $\mathrm{SiO}_{2}$ spheres on top of the solar cell. In Fig. 9(a) the case of small spheres with $60 \mathrm{~nm}$ radius is represented. A clear increase in quantum efficiency over almost the entire wavelength range is observed. This almost wavelength-independent behavior and the smoothening of interference fringes are in good agreement with the theoretically predicted antireflection effect. A total increase in short circuit current density of $2.1 \mathrm{~mA} / \mathrm{cm}^{2}$ was measured for this device with $460 \mathrm{~nm}$ absorber thickness.
The case of larger nanospheres was experimentally realized using $r=300 \mathrm{~nm}$ particles and is shown in Fig. 9(b). Around 500-600 and $900 \mathrm{~nm}$ wavelength a small increase is observed. As we have seen from the theoretical investigations, the various order Mie resonances and whispering gallery modes of the large nanospheres only occur in narrow wavelength bands. Since experimentally the perfect conditions of nanoparticle packing cannot be guaranteed, particularly the modes which rely on the nanoparticle interaction are hard to reveal. A larger benefit therefore clearly results from the small antireflection sphere arrays. Detailed experiments can also be found in Ref. 45.

Benefits of dielectric nanosphere coatings have been revealed e.g., in GaAs and Si solar cells. Whispering gallery modes, ${ }^{10}$ mixed graded index Mie-scattering ${ }^{46}$ and focusing behavior ${ }^{47}$ of nanoparticles have been identified to lead to enhanced incoupling of light. All these examples benefit from a close distance of the nanospheres to the absorber layer. Again, CIGSe solar cells with thicker front layers consisting of $\mathrm{AZO}, \mathrm{i}-\mathrm{ZnO}$, and $\mathrm{CdS}$ complicate coupling of light from resonant modes into the absorber. The increased spacing effectively makes only the antireflection behavior beneficial.

\section{Integration at back}

\section{a. Theoretical}

Placing the nanoparticles in close vicinity to the absorber layer has been identified as advantageous for enhancing the absorption in CIGSe and not in the contact layers. For the metallic nanoparticles this close contact to the absorber was complicated by diffusion of the nanoparticle material. Recombination of charge carriers at the metal surfaces was another drawback. Using metal nanoparticles wrapped in a dielectric shell was an option to overcome these challenges and still exploit the plasmonic enhancement properties. On the other hand, we can directly consider pure dielectric nanoparticles and exploit their photonic resonances. Due to the high thermal and
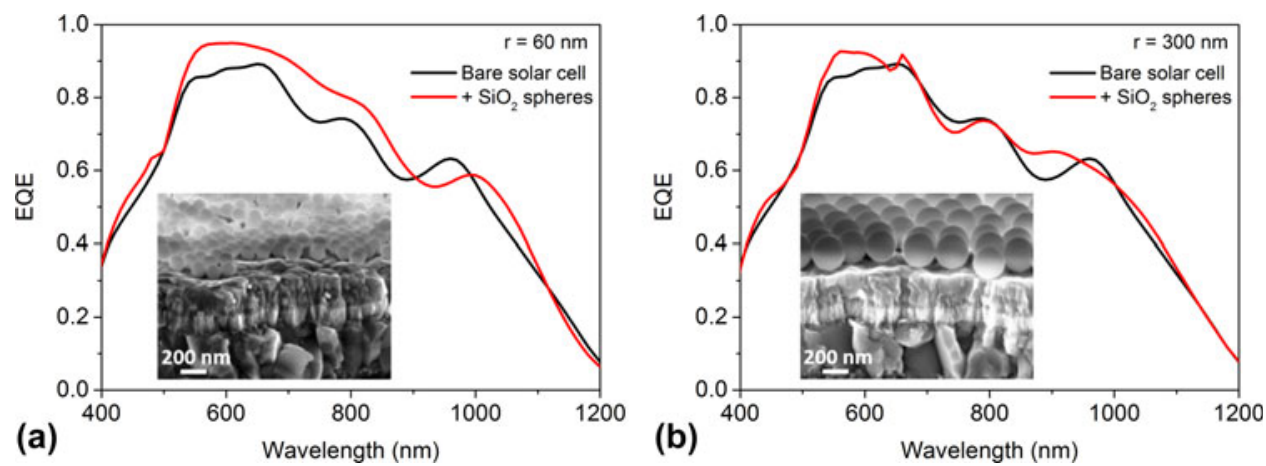

FIG. 9. Experimental integration of dielectric $\mathrm{SiO}_{2}$ nanoparticles at front (air/AZO) interface. $\mathrm{EQE}$ of ultrathin $\mathrm{CIGSe}$ solar cell with $\mathrm{SiO}{ }_{2}$ nanoparticles on top for (a) $r=60 \mathrm{~nm}$ and (b) $r=300 \mathrm{~nm}$ spheres as compared to the bare solar cell; insets show SEM cross-sections of the devices. ${ }^{45}$ 
electrical stability dielectric particles can be directly integrated underneath the absorber layer.

Figure 10(a) shows the absorption enhancement for $\mathrm{SiO}_{2}$ nanoparticles integrated at the CIGSe/Mo interface as a function of nanoparticle radius and pitch in multiple $m$ of diameter. Small spheres have a negligible effect, only starting from a radius of approximately $100 \mathrm{~nm}$ is absorption enhancement observed. The case of $r=250 \mathrm{~nm}$ and $m>1.5$ is represented in black in the figure since these parameter settings could not be calculated for an absorber layer of $400 \mathrm{~nm}$ thickness and considering volume compensation but not conformal overgrowth of CIGSe. A maximum enhancement of $3.6 \mathrm{~mA} / \mathrm{cm}^{2}$ is found for $r=225 \mathrm{~nm}$ and $m=1.25$ which corresponds to a total of $29.1 \mathrm{~mA} / \mathrm{cm}^{2}$ short circuit current density. Compared to the integration of $\mathrm{Ag}$ nanoparticles underneath ITO or coreshell nanoparticles on Mo, the enhancement is more than two times higher. Also the enhancement obtained by integrating dielectric nanoparticles at the front interface is significantly surpassed. In that latter case, the smaller nanoparticles performed best, acting as an effective antireflection coating. For the case of dielectric nanoparticles at the rear interface, reflection is enhanced for the small nanoparticles [see Fig. 10(b)], corresponding to a beneficial backscattering toward the absorber. In the range of highest absorption enhancement, reflection is however reduced, underlining the strong light trapping.

For the most promising multiple $m=1.25$, Fig. 10(c) depicts the wavelength dependent absorption in CIGSe for the three largest radii investigated $(200,225$, and $250 \mathrm{~nm})$. A strong enhancement in the entire wavelength range above $530 \mathrm{~nm}$ is observed as compared to the reference without integrated nanoparticles (gray). The case of a transparent back contact ( $250 \mathrm{~nm}$ ITO as previously) is depicted as well for the optimum $r=225 \mathrm{~nm}$ particles and in addition to an absorption enhancement of $3.6 \mathrm{~mA} / \mathrm{cm}^{2}$ a transmission corresponding to $3.1 \mathrm{~mA} / \mathrm{cm}^{2}$ can be gained here. In the absorption curves for integrated nanoparticles Fabry-Perot resonance peaks red-shift related to the compensated height of the CIGSe layer. On top of these peaks, at long wavelengths strongly pronounced resonances appear. Whereas the broadband enhancement above $530 \mathrm{~nm}$ can be linked to Mie resonances, the distinct peaks visible above $1100 \mathrm{~nm}$ are attributed to waveguide modes which result from the nanoparticle coupling. ${ }^{48}$ Figure $10(\mathrm{~d})$ reveals the electric field distributions at the peak positions. For the smallest shown particle $(r=200 \mathrm{~nm})$, the field enhancement on a scale from 0 to 3 is still weak, see the top left picture. For the $r=225 \mathrm{~nm}$ particle, the same mode (bottom left picture)
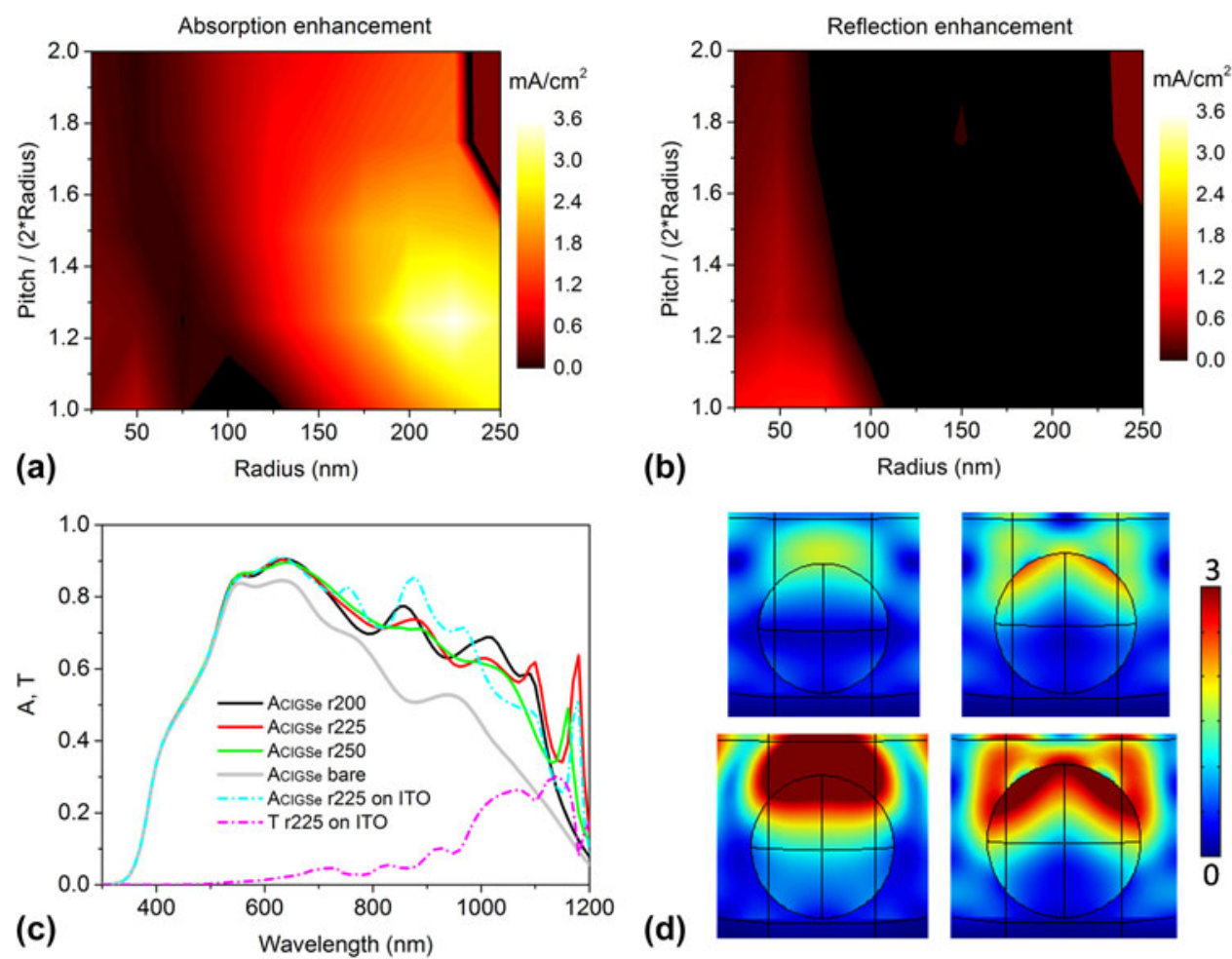

FIG. 10. Theoretical integration of dielectric $\mathrm{SiO}_{2}$ nanoparticles at back (CIGSe/Mo) interface. (a) Absorption enhancement in CIGSe as a function of radius $r$ and pitch over diameter (multiple $m$ ) and (b) corresponding reflection enhancement on the same scale; (c) absorption in CIGSe as a function of wavelength for the case of $m=1.25$ and radii 200, 225, and $250 \mathrm{~nm}$ spheres compared to the bare solar cell, also shown the case of $r=225 \mathrm{~nm}$ spheres on ITO and (d) electric field distributions around the spheres for $r$ and $\lambda$ equal to top left: 200 and $1090 \mathrm{~nm}$, top right: 225 and $1100 \mathrm{~nm}$, bottom left: 225 and $1180 \mathrm{~nm}$ and bottom right: 250 and $1160 \mathrm{~nm}$. 


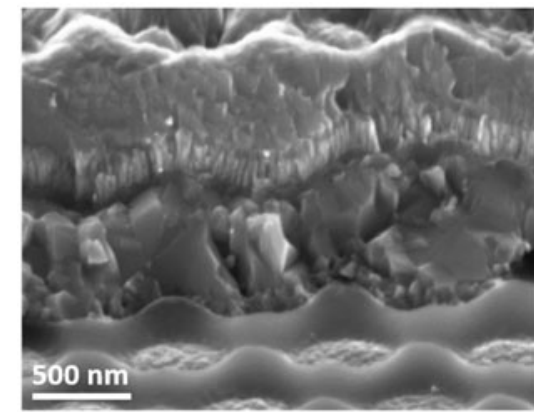

(a)

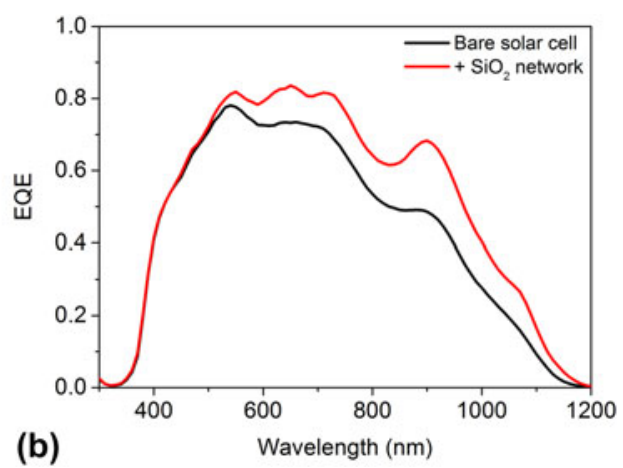

(b)

FIG. 11. Experimental integration of dielectric $\mathrm{SiO}_{2}$ nanopatterns at back (CIGSe/Mo) interface. (a) Cross-sectional SEM picture of ultrathin CIGSe solar cell with $\mathrm{SiO}_{2}$ network at the back contact (visible free-standing on Mo in the front); (b) broadband EQE enhancement by integration of the network.

leads to a huge field localization in the absorber, explaining the strong resonance peak at $\lambda=1180 \mathrm{~nm}$. For the same sphere, another resonance appears at $\lambda=1100 \mathrm{~nm}$ which is shown in the top right picture. This mode is the prominent one for the $r=250 \mathrm{~nm}$ sphere and becomes much more pronounced here as shown in the bottom right picture. The field localization either on top or at the side edges of the spheres is generally observed also for other shapes of dielectric nanoparticles integrated at the rear interface of an ultrathin CIGSe solar cell. For more details and the distribution of field intensities in case of hemispherical or tapered structures see Ref. 48.

\section{b. Experimental}

A spacing of approximately half the diameter of the dielectric spheres integrated at the rear interface has been shown to be the theoretical optimum. In contrast to the front interface, a closely packed sphere array would also not be electrically beneficial here since the rear contact would be isolated. Increasing the spacing between the spheres is thus crucial from an optical and an electrical point of view. Experimentally, the size of the PS spheres can easily be reduced by plasma etching in oxygen atmosphere. Yet, the PS spheres are not thermally stable and would not persist at the rear interface after the high temperature deposition process of CIGSe. $\mathrm{SiO}_{2}$ spheres therefore would be preferred, but in this case plasma etching generates greenhouse gasses making handling more complicated.

One solution is an alternative fabrication of the $\mathrm{SiO}_{2}$ nanoparticles, e.g., by nanoimprinting. For nanoimprinted dielectric patterns at the Mo/CIGSe interface, we have shown an increase in short-circuit current density of $2.3 \mathrm{~mA} / \mathrm{cm}^{2}$ and a related efficiency of $12.3 \%$ for ultrathin absorbers. ${ }^{49}$ But also the approach of nanosphere lithography offers an alternative option for fabrication of thermally stable dielectric nanostructures: the etched PS sphere template can serve as a mask for $\mathrm{SiO}_{2}$ deposition and after removal of the PS spheres a $\mathrm{SiO}_{2}$ network remains. The resulting network is depicted in Fig. 11(a) - free standing on Mo in the front and overcoated with the CIGSe solar cell at the back. For this example, PS spheres with a diameter of $909 \mathrm{~nm}$, which could be reduced to $630-640 \mathrm{~nm}$ by plasma etching for $12 \mathrm{~min}$ in $\mathrm{O}_{2}$ at a pressure of $0.2 \mathrm{mbar}$, were used as a mask for $\mathrm{SiO}_{2}$ deposition.

Figure 11(b) provides evidence for the benefits of integrating the $\mathrm{SiO}_{2}$ network at the rear of an ultrathin CIGSe solar cell. As can be seen from the quantum efficiency measurement, the absorption is significantly enhanced in the entire wavelength range from 500 to $1200 \mathrm{~nm}$. A related increase in short circuit current density from 23.8 to $27.7 \mathrm{~mA} / \mathrm{cm}^{2}$ leads to a relative increase in efficiency by $14 \%$. Compared to the theoretical predictions for the integration of $\mathrm{SiO}_{2}$ nanoparticles, the network does not exhibit sharp resonances above $1100 \mathrm{~nm}$ which can be related to different resonance conditions for particles and holes. However, the network configuration may provide an additional benefit over the particles: since a much larger back contact area is covered with isolating $\mathrm{SiO}_{2}$, point contacts are formed which can reduce rear surface recombination. ${ }^{50}$ Thus the structure is able to combine electrical and optical benefits.

Again, absorption enhancement by integration of dielectric nanoparticles at the rear interface has first been proven for Si solar cells. Dielectric nanoparticles acting as Mie scatterers ${ }^{9}$ or diffractive elements ${ }^{51}$ were reported. Since in the case of thermally and electrically stable dielectric nanoparticles we can also achieve direct integration underneath CIGSe, this is the most promising configuration for chalcopyrite-based solar cells. We could successfully prove significant absorption enhancement ${ }^{49}$ and additionally identify electrical benefits of the nanostructures at the back contact. ${ }^{48}$

\section{SUMMARY}

In this paper, we have investigated metallic, metaldielectric core-shell, and dielectric nanoparticles integrated 
either at the front or the rear interfaces of an ultrathin CIGSe solar cell. The example calculations were assuming spherical nanoparticles which may not necessarily correspond to the realistic situation. Therefore, absolute values of absorption enhancement for the various configurations given here should be used for comparative purpose only and final enhancement benchmarked against maximum expected values for the respective experimental geometries. In the following, we summarize the major benefits and drawbacks of the different configurations.

Ag nanoparticles at the front air/AZO interface provide a strong forward scattering which can be theoretically exploited to reduce reflection of the device. Yet, the metallic nanoparticles also show parasitic absorption which is strongly pronounced for small particles and can be mostly suppressed for radii larger than $75 \mathrm{~nm}$. The scattering into a large angular range is another characteristic of the metallic nanoparticles, which is however attenuated due to the front contact layers separating the nanoparticles from the absorber. Losses in contact layers are a general drawback when nanoparticles are not directly touching the absorber. The maximum theoretical enhancement found was $1.2 \mathrm{~mA} / \mathrm{cm}^{2}$. Experimentally, particle integration at the front avoids interfering with device processes. In the case of metallic nanoparticles, annealing steps have to be considered carefully due to the temperature impact on the device. It is found that in the experiment Ag nanoparticles on top of CIGSe solar cells generally increase the reflection rather than reduce it.

$\mathrm{Ag}$ nanoparticles at the rear (ITO/glass) interface are a promising alternative to suppress parasitic absorption in the first place and exploit backscattering of the metallic nanoparticles toward the high index absorber layer. Since thermally and electrically passivating layers are required between the nanoparticles and the absorber, there is only a limited exploitation of scattering and near-field effects and losses in contacts need to be accepted. Integration in a device with a transparent back contact is also beneficial from the perspective of reduced absorption in Mo. The maximum theoretical enhancement found was $2.2 \mathrm{~mA} / \mathrm{cm}^{2}$ plus $2.0 \mathrm{~mA} / \mathrm{cm}^{2}$ transmission. Experimentally, a thermal passivation of Ag nanoparticles can be achieved by ALD $\mathrm{Al}_{2} \mathrm{O}_{3}$. Remaining challenges lie in the mechanically stable contact formation with integrated nanoparticles.

Ag@dielectric core-shell nanoparticles can be directly integrated underneath the absorber layer. A high refractive index shell material is required to avoid trapping light in the shell. In the case of a refractive index equal to or higher than the surrounding, a shell thickness of $10 \mathrm{~nm}$, which is expected to provide passivation, is acceptable. Scattering and near-fields are then directly exploited in the absorber layer. In the case of a transparent back contact, absorption enhancement and transmission can be flexibly balanced using the nanoparticles. The maximum theoretical enhancement found was $1.9 \mathrm{~mA} / \mathrm{cm}^{2}$ plus $2.7 \mathrm{~mA} / \mathrm{cm}^{2}$ transmission. Experimentally, thermal stabilization can be achieved for metal-dielectric core-shell nanoparticles underneath the absorber layer. The challenge lies in fabricating adequately sized (radii larger $75 \mathrm{~nm}$ ) core-shell particles with a high refractive index shell material.

$\mathrm{SiO}_{2}$ nanoparticles at the front (air/AZO) interface are able to enhance light incoupling without parasitic absorption in the nanoparticles. The antireflection effect of the small nanospheres (radii around $75 \mathrm{~nm}$ ) is most pronounced and can outperform a planar antireflection layer in terms of increased acceptance angle of incident light. For larger spheres (e.g., $225 \mathrm{~nm}$ radius) Mie resonances and whispering gallery modes dominate, which show a spectrally confined and thus weaker overall enhancement. Losses in the contact layers need to be considered here as well. The maximum theoretical enhancement found was $1.6 \mathrm{~mA} / \mathrm{cm}^{2}$. Experimentally, the deposition of dielectric particles on top of the finished solar cell is straight forward and the related chemical processes are compatible with CIGSe solar cells. Enhancement in line with the theoretical predictions could be demonstrated.

$\mathrm{SiO}_{2}$ nanoparticles at the rear (CIGSe/Mo) interface provide the strongest light interaction and resonances leading to efficient light trapping. The direct contact to the absorber layer, feasible for these electrically and thermally passivated nanoparticles, proves to be highly beneficial. The light collected by the nanoparticles can be directly coupled to the absorber layer. The structures also benefit from a regular arrangement of the nanoparticles with close spacing and thus nanoparticle coupling. The maximum theoretical enhancement found was $3.6 \mathrm{~mA} / \mathrm{cm}^{2}$ and in the case of a transparent back contact, an additional $3.1 \mathrm{~mA} / \mathrm{cm}^{2}$ transmission. Experimentally, the dielectric nanostructures can be successfully integrated in the solar cell and a conformal overgrowth with the absorber layer achieved. $3.9 \mathrm{~mA} / \mathrm{cm}^{2}$ enhancement in short circuit current density for a dielectric network on Mo is shown in this paper.

Compared to other solar cell technologies (e.g., Si, GaAs, or organic absorber materials), CIGSe solar cells pose particular challenges for the effective integration of nanostructures for absorption enhancement. The high temperature absorber deposition process requires not just electrical but also thermal passivation for rear interface integration. Much thicker contact and additional passivation layers complicate placing the nanoparticles in close vicinity to the absorber, which however is of utmost importance for effective enhancement. Therefore, directly integrated, stable dielectric nanoparticles have proven most beneficial and gave the best improvement.

Looking beyond just optical enhancement, dielectric nanostructures have also been identified to provide back surface passivation. Point contact structures have been proposed in Ref. 50 and the combined optical and 
electrical benefit of dielectric nanoparticles was just recently proven in Ref. 48. Transparent back contacts are the key to most efficient light exploitation and the nanoparticles allow for a wavelength dependent balance between absorption and transmission which is of high interest for semitransparent devices.

In total, metallic nanoparticles are beneficial for their scattering toward high-index materials and into large angles, but suffer from parasitic absorption and lack of electrical and thermal passivation. Metal-dielectric core-shell nanoparticles can be a way to confront the passivation challenge and provide efficient enhancement when integrated into the absorber. Generally, any separation layer between nanostructures and absorber should be avoided to minimize losses. Dielectric nanoparticles have proven to be most beneficial, due to their high stability and strong resonant modes. Furthermore, they enable a combined optical-electrical benefit, which will be a focus of future investigations.

\section{ACKNOWLEDGMENTS}

The authors would like to thank S. Duan for absorber and C. Ferber \& M. Kirsch for front contact fabrication, K. Harbauer for deposition of sputtered and M. Latzel \& M. Goebelt for ALD $\mathrm{Al}_{2} \mathrm{O}_{3}$. A. Steigert is greatly acknowledged for performing XPS measurements. The work received funding from the Helmholtz Association for the Young Investigator Group VH-NG-928 within the Initiative and Networking Fund.

\section{REFERENCES}

1. Press Release 09/2016: ZSW Sets New World Record for Thin-film Solar Cells. https://www.zsw-bw.de/en/newsroom/news/newsdetail/news/detail/News/zsw-sets-new-world-record-for-thin-film-solar-cells.html (2016).

2. C. Morioka, K. Shimazaki, S. Kawakita, M. Imaizumi, H. Yamaguchi, T. Takamoto, S-Î. Sato, T. Ohshima, Y. Nakamura, K. Hirako, and M. Takahashi: First flight demonstration of film-laminated InGaP/GaAs and CIGS thin-film solar cells by JAXA's small satellite in LEO. Prog. Photovoltaics Res. Appl. 19(7), 825 (2011).

3. British Geological Survey: Risk List. http://www.bgs.ac.uk/mineralsuk/ statistics/risklist.html (2015).

4. J. Palm, F. Karg, H. Schneider, K. Kushiya, L. Stolt, A.N. Tiwari, E. Niemi, M. Beck, C. Eberspacher, P. Wohlfart, A. Bayman, U. Schoop, B. Wieting, K. Ramanathan, B. Dimmler, C. Kuhn, S. Whitelegg, U. Rühle, D. Lincot, N. Naghavi, T. Walter, R. Schlatmann, M. Lux-Steiner, A. Kuypers, B. Szyszka, S. Siebentritt, P. Lechner, M. Powalla, R. Noufi, and H.W. Schock: White paper for CIGS thin film solar cell technology. http://cigs-pv.net/ (2015).

5. M. Gu, Z. Ouyang, B. Jia, N. Stokes, X. Chen, N. Fahim, X. Li, M.J. Ventura, and Z. Shi: Nanoplasmonics: A frontier of photovoltaic solar cells. Nanophotonics 1(3-4), 235 (2012).

6. S. Pillai, K.R. Catchpole, T. Trupke, and M.A. Green: Surface plasmon enhanced silicon solar cells. J. Appl. Phys. 101(9), 093105/1 (2007).

7. H.A. Atwater and A. Polman: Plasmonics for improved photovoltaic devices. Nat. Mater. 9, 205 (2010).
8. A. Ott, S. Ring, G. Yin, W. Calvet, B. Stannowski, Y. Lu, R. Schlatmann, and M. Ballauff: Efficient plasmonic scattering of colloidal silver particles through annealing-induced changes. Nanotechnology 25, 455706 (2014).

9. M. van Lare, F. Lenzmann, and A. Polman: Dielectric back scattering patterns for light trapping in thin-film Si solar cells. Opt. Express 21(18), 20738 (2013).

10. J. Grandidier, R.A. Weitekamp, M.G. Deceglie, D.M. Callahan, C. Battaglia, C.R. Bukowsky, C. Ballif, R.H. Grubbs, and H.A. Atwater: Solar cell efficiency enhancement via light trapping in printable resonant dielectric nanosphere arrays. Phys. Status Solidi A 210(2), 255 (2013).

11. M. Schmid, S. Tsakanikas, G. Mangalgiri, P. Andrae, M. Song, G. Yin, W. Riedel, and P. Manley: Nano-optical concept design for light management. In Proc. SPIE 9626, Optical Systems Design 2015. Optical Design and Engineering VI, Vol. 9626, 2015; p. 96260E.

12. http://www.jcmwave.com/jcmsuite.

13. https://www.comsol.de/comsol-multiphysics.

14. G. Yin, P. Manley, and M. Schmid: Influence of substrate and its temperature on the optical constants of $\mathrm{CuIn}_{1-x} \mathrm{Ga}_{x} \mathrm{Se}_{2}$ thin films. J. Phys. D: Appl. Phys. 47(13), 135101 (2014).

15. http://www.helmholtz-berlin.de/forschung/oe/ee/nanooptix/refdex/ index_en.html.

16. E.D. Palik: Handbook of Optical Constants of Solids (Academic Press, Cambridge, 1985).

17. G. Yin, V. Brackmann, V. Hoffmann, and M. Schmid: Enhanced performance of ultra-thin $\mathrm{Cu}(\mathrm{In}, \mathrm{Ga}) \mathrm{Se}_{2}$ solar cells deposited at low process temperature. Sol. Energy Mater. Sol. Cells 132, 142 (2015).

18. A. Kosiorek, W. Kandulski, P. Chudzinski, K. Kempa, and M. Giersig: Shadow nanosphere lithography: Simulation and experiment. Nano Lett. 4(7), 1359 (2004).

19. G.D. Moon, T.I. Lee, B. Kim, G. Chae, J. Kim, S. Kim, J-M. Myoung, and U. Jeong: Assembled monolayers of hydrophilic particles on water surfaces. ACS Nano 5(11), 8600 (2011).

20. http://microparticles.de/.

21. S. Wu, A.W. Schell, M. Lublow, J. Kaiser, T. Aichele, S. Schietinger, F. Polzer, S. Kühn, X. Guo, O. Benson, M. Ballauff, and Y. Lu: Silica-coated Au/Ag nanorods with tunable surface plasmon bands for nanoplasmonics with single particles. Colloid Polym. Sci. 291(3), 585 (2013).

22. I. Gorelikov and N. Matsuura: Single-step coating of mesoporous silica on cetyltrimethyl ammonium bromide-capped nanoparticles. Nano Lett. 8(1), 369 (2008).

23. W. Stöber, A. Fink, and E. Bohn: Controlled growth of monodisperse silica spheres in the micron size range. J. Colloid Interface Sci. 26(1), 62 (1968).

24. P.R. West, S. Ishii, G.V. Naik, N.K. Emani, V.M. Shalaev, and A. Boltasseva: Searching for better plasmonic materials. Laser Photonics Rev. 4(6), 795 (2010).

25. P. Manley, S. Burger, F. Schmidt, and M. Schmid: Design principles for plasmonic nanoparticle devices. In Progress in Nonlinear Nano-optics, S. Sakabe, C. Lienau, and R. Grunwald eds.; Springer International Publishing, Berlin, 2015; p. 223.

26. C.F. Bohren and D.R. Huffmann: Absorption and scattering of light by small particles (Wiley-VCH, New York, 1998).

27. G. Yin: Preparation of Ultra-thin CuIn ${ }_{1-\mathrm{x}} \mathrm{Ga} a_{\mathrm{x}} \mathrm{Se}_{2}$ Solar Cells and Their Light Absorption Enhancement (TU Berlin, Berlin, 2015).

28. D. Derkacs, S.H. Lim, P. Matheu, W. Mar, and E.T. Yu: Improved performance of amorphous silicon solar cells via scattering from surface plasmon polaritons in nearby metallic nanoparticles. Appl. Phys. Lett. 89(9), 093103/1 (2006).

29. K. Nakayama, K. Tanabe, and H.A. Atwater: Plasmonic nanoparticle enhanced light absorption in GaAs solar cells Appl. Phys. Lett. 93(12), 121904/1 (2008). 
30. J. Mertz: Radiative absorption, fluorescence, and scattering of a classical dipole near a lossless interface: A unified description. J. Opt. Soc. Am. B 17(11), 1906 (2000).

31. J.H. Boyle, B.E. McCandless, G.M. Hanket, and W.N. Shafarman: Structural characterization of the $(\mathrm{AgCu})(\mathrm{InGa}) \mathrm{Se}_{2}$ thin film alloy system for solar cells. Thin Solid Films 519(21), 7292 (2011).

32. G. Yin, A. Steigert, P. Andrae, M. Goebelt, M. Latzel, P. Manley, I. Lauermann, S. Christiansen, and M. Schmid: Integration of plasmonic $\mathrm{Ag}$ nanoparticles as a back reflector in ultra-thin $\mathrm{Cu}(\mathrm{In}, \mathrm{Ga}) \mathrm{Se}_{2}$ solar cells. Appl. Surf. Sci. 355, 800 (2015).

33. Y. Yang, S. Pillai, H. Mehrvarz, H. Kampwerth, A. Ho-Baillie, and M.A. Green: Enhanced light trapping for high efficiency crystalline solar cells by the application of rear surface plasmons. Sol. Energy Mater. Sol. Cells 101, 217 (2012).

34. H. Tan, R. Santbergen, A.H. Smets, and M. Zeman: Plasmonic light trapping in thin-film silicon solar cells with improved self-assembled silver nanoparticles. Nano Lett. 12(8), 4070 (2012).

35. V.E. Ferry, M.A. Verschuuren, H.B.T. Li, E. Verhagen, R.J. Walters, R.E.I. Schropp, H.A. Atwater, and A. Polman: Light trapping in ultrathin plasmonic solar cells. Opt. Express 18(S2), A237 (2010).

36. P. Manley, F. Schmidt, and M. Schmid: Light extraction from plasmonic particles with dielectric shells and overcoatings. In Renewable Energy and the Environment: Optical Nanostructures and Advanced Materials for Photovoltaics (Optical Society of America, Tucson 2013); PW3B.7.

37. B. Chen, W. Zhang, X. Zhou, X. Huang, X. Zhao, H. Wang, M. Liu, Y. Lu, and S. Yang: Surface plasmon enhancement of polymer solar cells by penetrating $\mathrm{Au} / \mathrm{SiO}_{2}$ core/shell nanoparticles into all organic layers. Nano Energy 2(5), 906 (2013).

38. Q. Xu, F. Liu, W. Meng, and Y. Huang: Plasmonic core-shell metal-organic nanoparticles enhanced dye-sensitized solar cells. Opt. Express 20(S6), A898 (2012).

39. M. Saliba, W. Zhang, V.M. Burlakov, S.D. Stranks, Y. Sun, J.M. Ball, M.B. Johnston, A. Goriely, U. Wiesner, and H.J. Snaith: Plasmonic-induced photon recycling in metal halide perovskite solar cells. Adv. Funct. Mater. 25(31), 5038 (2015).

40. M. Schmid, P. Andrae, and P. Manley: Plasmonic and photonic scattering and near fields of nanoparticles. Nanoscale Res. Lett. $\mathbf{9}$, $50 / 1$ (2014).
41. Y.A. Akimov, W.S. Koh, S.Y. Sian, and S. Ren: Nanoparticleenhanced thin film solar cells: Metallic or dielectric nanoparticles? Appl. Phys. Lett. 96(7), 073111 (2010).

42. J. van de Groep and A. Polman: Designing dielectric resonators on substrates: Combining magnetic and electric resonances. Opt. Express 21(22), 26285 (2013).

43. M. Schmid and P. Manley: Nano- and microlenses as concepts for enhanced performance of solar cells. J. Photonics Energy 5(1), 057003 (2014).

44. A.N. Oraevsky: Whispering-gallery waves. Quantum Electron. 32 (5), 377 (2002).

45. G. Yin, P. Manley, and M. Schmid: Light absorption enhancement for ultra-thin $\mathrm{Cu}\left(\mathrm{In}_{1-x} \mathrm{Ga}_{x}\right) \mathrm{Se}_{2}$ solar cells using closely packed 2-D $\mathrm{SiO}_{2}$ nanosphere arrays. Sol. Energy Mater. Sol. Cells 153, 124 (2016).

46. A. Lin, Y-K. Zhong, and S-M. Fu: The versatile designs and optimizations for cylindrical $\mathrm{TiO}_{2}$-based scatterers for solar cell anti-reflection coatings. Opt. Express 21(S6), A1052 (2013).

47. C.R. Simovski, A.S. Shalin, P.M. Voroshilov, and P.A. Belov: Photovoltaic absorption enhancement in thin-film solar cells by non-resonant beam collimation by submicron dielectric particles. J. Appl. Phys. 114(10), 103104 (2013).

48. G. Yin, M. Knight, M-C. van Lare, A. Polman, and M. Schmid: Opto-electronic enhancement of ultrathin $\mathrm{Cu}(\mathrm{In}, \mathrm{Ga}) \mathrm{Se}_{2}$ solar cells by nanophotonic contacts. Adv. Opt. Mat., accepted (2016).

49. C. van Lare, G. Yin, A. Polman, and M. Schmid: Light coupling and trapping in ultrathin $\mathrm{Cu}(\mathrm{In}, \mathrm{Ga}) \mathrm{Se}_{2}$ solar cells using dielectric scattering patterns. ACS Nano 9(10), 9603 (2015).

50. B. Vermang, J.T. Wätjen, V. Fjällström, F. Rostvall, M. Edoff, R. Gunnarsson, I. Pilch, U. Helmersson, R. Kotipalli, F. Henry, and D. Flandre: Highly reflective rear surface passivation design for ultra-thin $\mathrm{Cu}(\mathrm{In}, \mathrm{Ga}) \mathrm{Se}_{2}$ solar cells. Thin Solid Films 582, 300 (2015).

51. E-C. Wang, S. Mokkapati, T.P. White, T. Soderstrom, S. Varlamov, and K.R. Catchpole: Light trapping with titanium dioxide diffraction gratings fabricated by nanoimprinting. Prog. Photovoltaics Res. Appl. 22(5), 587 (2014). 